\title{
Complex Permittivity and Permeability of Composite Materials Based on Carbonyl Iron Powder Over an Ultrawide Frequency Band
}

\author{
Vladimir N. Semenenko, ${ }^{1}$ Vladimir A. Chistyaev, ${ }^{1}$ Alexey A. Politiko $\odot,{ }^{1}$ Sergey G. Kibets, ${ }^{1}$ \\ Vladimir N. Kiselø, ${ }^{1}$ Cameron P. Gallagher, ${ }^{2}$ Conor McKeever $\odot,{ }^{2,3, *}$ Alastair P. Hibbins $\odot,{ }^{2}$ \\ Feodor Y. Ogrin, ${ }^{2,3}$ and J. Roy Sambles ${ }^{2}$ \\ ${ }^{1}$ Institute for Theoretical and Applied Electromagnetics RAS, 125412, Moscow, Russian Federation \\ ${ }^{2}$ Department of Physics and Astronomy, University of Exeter, Exeter EX4 4QL, United Kingdom \\ ${ }^{3}$ MaxLLG Ltd, Exeter Science Park, Exeter EX5 2FN, United Kingdom
}

(Received 8 September 2020; revised 2 March 2021; accepted 16 June 2021; published 27 July 2021)

\begin{abstract}
The complex electrical permittivity and magnetic permeability of composite materials made of a polymer binder filled with micron-scale carbonyl iron powder (CIP) are measured between 0.1 and $39 \mathrm{GHz}$. Permeability is measured in overlapping frequency subbands using two different techniques: a free-space method from 3 to $39 \mathrm{GHz}$ and a coaxial impedance cell from 0.1 to $5 \mathrm{GHz}$. The dependence on filler concentration is studied for composites based on phosphated CIP R-100F-2. It is found that the static permittivity and permeability of the composites increase logarithmically with increasing percentage of CIP volume loading; this corresponds to Lichtenecker's law for a mixture of two components. It is demonstrated that by using the R-100F-2 type CIP it is possible to produce single-layer radar-absorbing materials with a relatively small thickness (less than $1.5 \mathrm{~mm}$ ) and a deep and broad normal-incidence reflectivity minimum (less than $-20 \mathrm{~dB}$ ) from 10 to $30 \mathrm{GHz}$.
\end{abstract}

DOI: 10.1103/PhysRevApplied.16.014062

\section{INTRODUCTION}

Microwave composites and metamaterials have been the focus of significant interest in recent years driven by the increasing demand for highly effective electromagnetic absorbing materials for use with modern radar and electronics technology [1-4]. Composite materials with controlled complex electrical permittivity and magnetic permeability are of considerable technological importance for antenna miniaturization [5], magnetic sensors [6], nonreciprocal devices [7], and microwave absorbers [8]. A variety of metallic magnetic materials have been successfully fabricated to increase the complex permeability of composites, including ferromagnetic microwires [9], electroplated wires [10], flakelike particles with large planar anisotropy [11,12], and core-shell structures with tunable magnetic and dielectric loss mechanisms [13-15]. Obviously, the focused synthesis and design of such materials and coatings cannot be achieved without characterization of their electrodynamic parameters over the frequency band of interest and, in general, the wider this band is the better.

Microwave absorption can be achieved using two loss mechanisms, namely, dielectric loss and magnetic loss. Due to their large complex permeability, magnetic

\footnotetext{
*c.mckeever@maxllg.com
}

absorbers are used at lower frequencies, which lead to excellent absorption properties. However, the complex permeability is known to decrease with increasing frequency, in accordance with Snoek's law [16]. This restricts the use of magnetic absorbers in the higher gigahertz bands. Dielectric absorbers are used in higher-frequency ranges, but they require high matching thicknesses, which limit their practical application [17,18]. Ferromagnetic multilayers have been intensively studied for both fundamental research and for application in microwave devices [19-21]. In terms of device applications, having a multilayer instead of just a monolayer provides more degrees of freedom for tailoring the intrinsic electromagnetic (EM) properties. Significant effort has been devoted to the development of multilayered structures incorporating ferromagnetic layers, such as hollow reduced graphene oxide microspheres embedded with Co nanoparticles [22], cobalt/polypyrrole (Co/PPy) nanocomposites [23], and $\mathrm{CoNi} / \mathrm{TiO}_{2}$ microspheres with wideband absorption properties [24]. These multicomponent structures can achieve tunable reflection loss and improved impedance matching by tailoring the parameters of the magnetic and dielectric layers.

The ideal microwave-absorbing material should have low density, small thickness, good chemical stability, and strong EM absorption across a wide-frequency band. Currently, many ways to develop radar-absorbing materials 


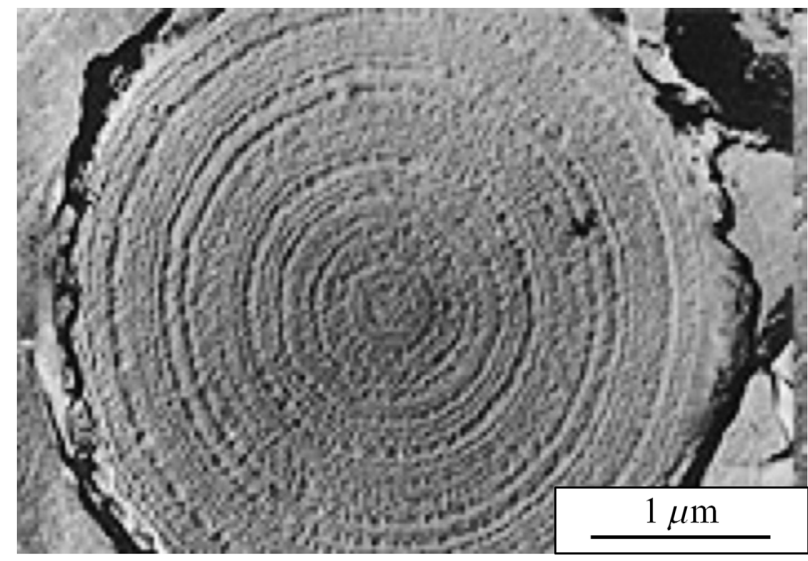

FIG. 1. Microphotography of the microscopic section of a carbonyl iron grain [26].

(RAMs) and coatings are known. For example, RAMs based on ferrites (ferrite-garnets, ferrite-spinels, hexaferrites, etc.) are designed for meter and decimeter wavelengths. The value of the relative magnetic permeability of such materials can vary from tens to hundreds in the long-wave region of the spectrum, which is especially important for the development of RAMs with small thicknesses at megahertz frequencies. However, ferrites suffer from having a decreasing dependence of the effective magnetic permeability with increasing frequency, according to Snoek's law $[16,25]$, which is given by

$$
\mu=1+\frac{\gamma 4 \pi M_{s}}{f}
$$

where $\gamma$ is the gyromagnetic ratio $(\gamma=2.8 \mathrm{GHz} / \mathrm{kOe}), M_{S}$ is the saturation magnetization of the material, and $f$ is the operating frequency. This present study concerns the frequency dependence of both complex electrical permittivity and magnetic permeability of composite materials with magnetic fillers based on different brands of carbonyl iron powder (CIP) produced by Sintez-CIP Ltd. (Russian Federation) and BASF SE (Germany). Measurements of material parameters of the composites are carried out from 0.1 to $39 \mathrm{GHz}$ using free-space and coaxial techniques. Special attention is paid to the study of the dependence of permittivity and permeability on the concentration of the R-100F-2 brand CIP embedded into a polymer matrix.

The feedstock carbonyl iron (hard) powder consists of spherical particles with an "onion-skin" layered structure (Fig. 1). This is obtained by thermal decomposition at temperatures of $200-250^{\circ} \mathrm{C}$ and a pressure of $150 \mathrm{~atm}$ of $\mathrm{Fe}(\mathrm{CO})_{5}$ (iron pentacarbonyl) with subsequent refining in hydrogen [26].

\section{COMPOSITE SAMPLES}

Thin sheets of materials made of two-component composites are used as samples for the measurements. The (a)

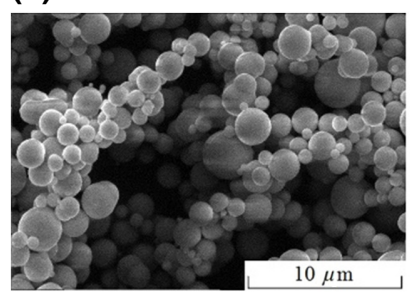

(c)

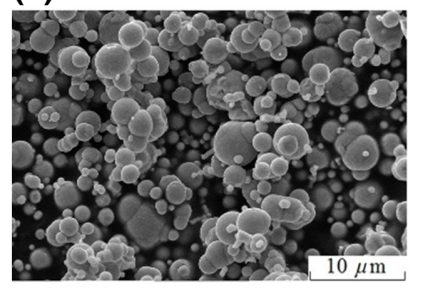

(b)

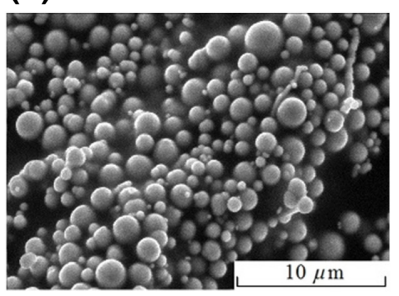

(d)

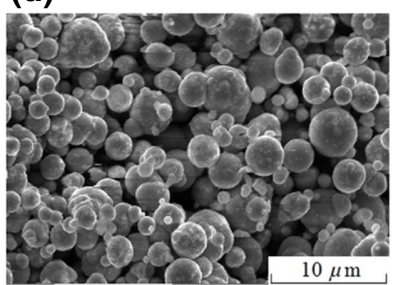

FIG. 2. SEM images of CIP particles of the following brands: (a) R-100F-2, (b) HL, (c) EN, (d) EW.

initial composite is a homogeneous highly viscous suspension of soft magnetic filler in the form of CIP introduced into a solution of an organosilicon polymer. Samples are made by air-spray paint technology. This production method allows one to readily increase the number of layers of material. At the same time, it is relatively easy to make samples with a uniform thickness over an area of many $\mathrm{cm}^{2}$, which helps to minimize measurement error of the material's parameters.

Morphology, microstructure, and particle size distribution of CIP are investigated by means of scanning electron

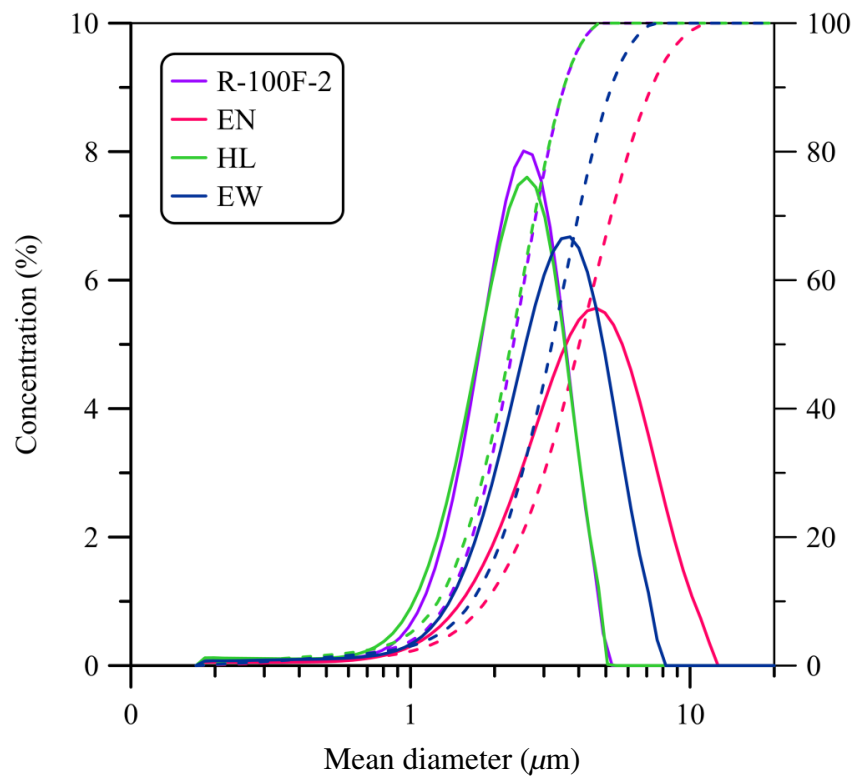

FIG. 3. Size distribution of CIP particles of R-100F-2, EN, $\mathrm{HL}$, and EW. 


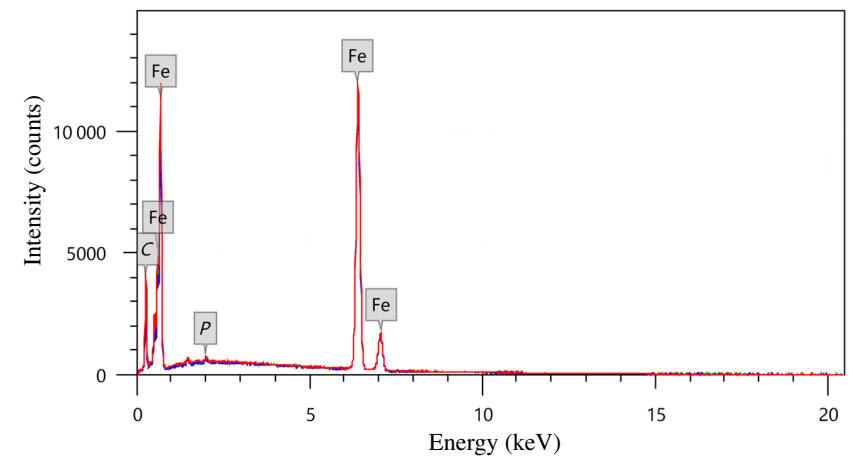

FIG. 4. EDX analysis of CIP R-100F-2.

microscopy (SEM). Photomicrographs of micron-sized particles of CIP R-100F-2, EN, HL, and EW, obtained using a Zeiss LEO 982 microscope, are presented in Fig. 2. These photographs reveal that the particles of CIP constitute granules of approximately the same near-spherical shape with a limited range of diameters.

For a more precise evaluation of the average particle size, a granulometric analysis of the fractional composition of the powders is carried out using a Fritch Analysette 22 laser diffractometer. From the distribution curves shown in Fig. 3, it can be seen that the particle size distribution functions have a Gaussian form with a maximum corresponding to the average particle diameter. This varies from 2 to $5 \mu \mathrm{m}$, depending on the type of CIP.

The performed granulometric analysis shows that the average particle size generally corresponds to data declared by the manufacturers. It is worth mentioning that the powders R-100F-2 and HL are almost identical in their fractional compositions.

Structural characterization results for CIP, obtained by energy-dispersive x-ray spectroscopy (EDX) of CIP R100F-2 using a SEM JCM-7000 instrument, are presented in Fig. 4. These results show that the volume loading of $\mathrm{Fe}$ in the powder is about $86 \%$, the volume loading of $\mathrm{P}$ does not exceed $0.3 \%$, and the volume loading of $\mathrm{C}$ is about $13.7 \%$.

\section{MEASUREMENT TECHNIQUES AND EXPERIMENTAL DATA}

The static magnetic properties of composite samples comprising of R-100F-2 CIP with different volume concentrations of powders are measured with a vibrating sample magnetometer (Fig. 5). All measurements are carried out at room temperature in the range of applied field from -15 to $15 \mathrm{kOe}$. All composite samples exhibit excellent soft magnetic properties with a coercivity $\left(H_{c}\right)$ of less than 15 Oe.

The saturation magnetization, $4 \pi M_{s}$, of the CIP composite samples increases linearly from 0.095 to $0.652 \mathrm{~T}$ with an increase in the volume loading of the CIP powder

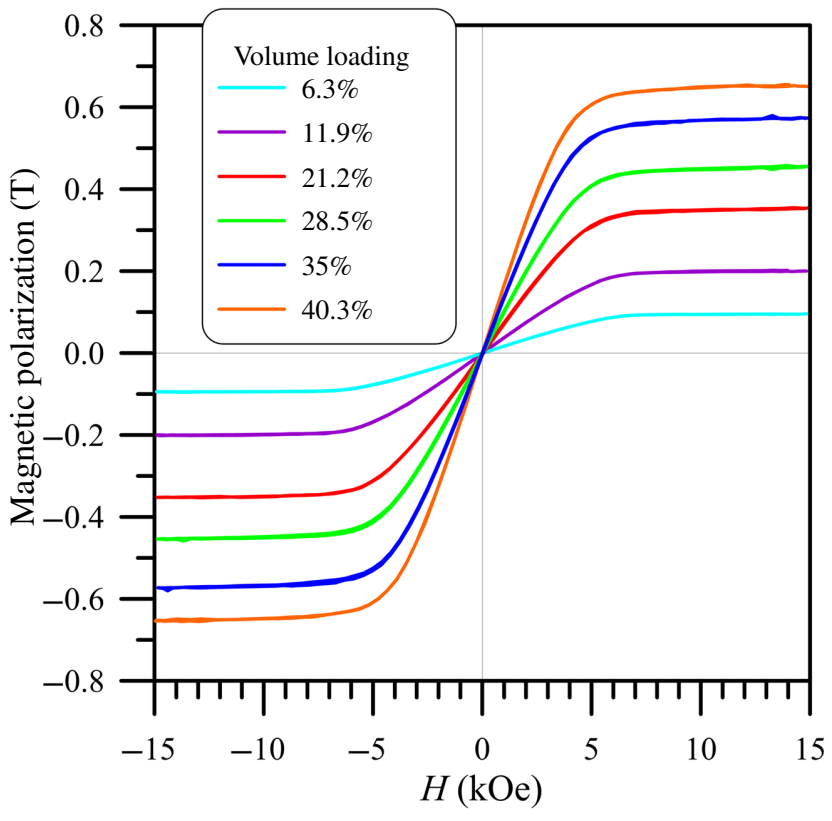

FIG. 5. Hysteresis loops of R-100F-2 composite samples for different volume loadings of CIP.

from 6.3 to $40.3 \%$, as shown in Fig. 6, where the points are experimental data and the solid line is a linear fit. The dashed line in Fig. 6 is the calculated saturation magnetization, $4 \pi M_{s}$, for composites loaded with pure Fe particles extracted using $4 \pi M_{s}=2.15 \mathrm{~T}$ [27] for pure bulk Fe. A comparison of saturation magnetization of pure $\mathrm{Fe}$ and R-100F2 CIP leads to the conclusion that R-100F-2 CIP

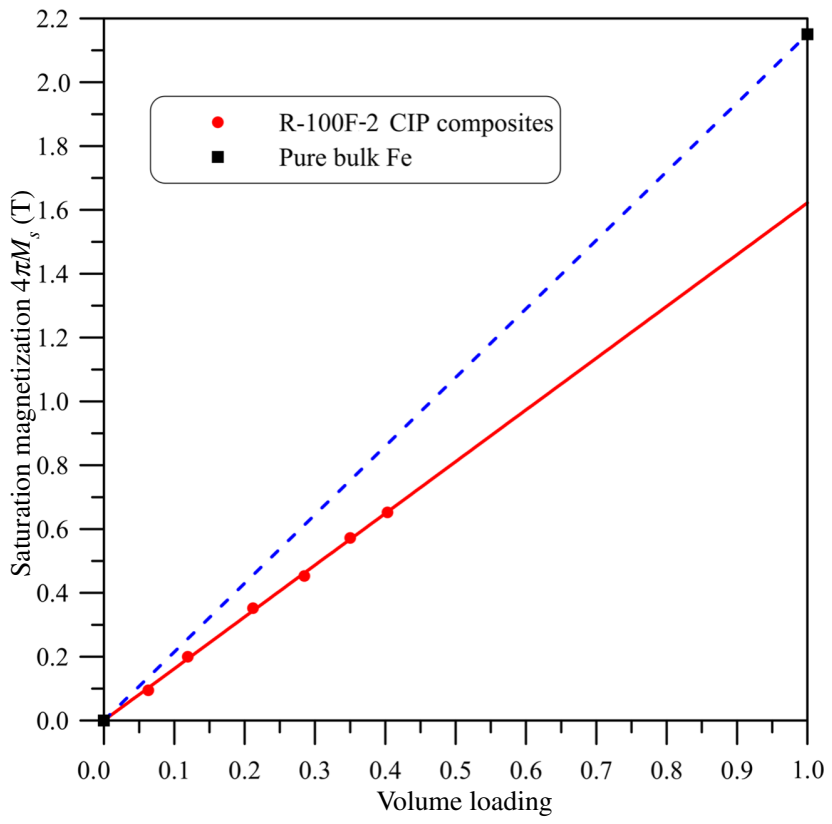

FIG. 6. Saturation magnetization of R-100F-2 CIP composites versus volume loading of CIP. 
has approximately $67 \%$ in the magnetic phase and $33 \%$ in the nonmagnetic phase (phosphate shells with carbides and nitrides of $\mathrm{Fe}$ in the onion boundary layers).

The complex electrical permittivity and magnetic permeability of composites are measured using two fundamentally different techniques: a coaxial cell and free space. The frequency range of measurements consists of three overlapping subbands: $0.1-5,3-24$, and 22-39 GHz.

Measurements of material parameters of the composites in free space are carried out over the two frequency subbands 3-24 and 22-39 GHz. One of the key features of this technique is the use of circular metal diaphragms with specific RAMs as a part of the measurement bench [8]. Thin films of sheet composite materials with linear dimensions of $200 \times 200 \mathrm{~mm}^{2}$ and $0.5 \mathrm{~mm}$ in thickness are used as the samples. To determine the permittivity and permeability of these samples, two parameters are measured: the normal-incidence transmission coefficient $\left(S_{21}\right)$ of a sample backed by a matched load and the reflection coefficient of a metal-backed sample $\left(S_{11}^{M}\right)$. The values of the complex reflection and transmission coefficients of a magnetodielectric sample at normal incidence of a plane electromagnetic wave (EMW) are expressed by the commonly used Fresnel formula [28]:

$$
S_{11}^{M}=\frac{x}{y}, \quad S_{21}=\frac{x+y}{1-y^{2}},
$$

where $x=i Z \sin \varphi+\cos \varphi ; y=i Z \sin \varphi-\cos \varphi ; Z=$ $\sqrt{\mu / \varepsilon}$ is the wave impedance of the sample; $\varphi=k d \sqrt{\varepsilon \mu}$ is the phase advance while an EMW travels through a sample; $k=2 \pi \sqrt{\varepsilon \mu} / \lambda$ is the wave number, where $\lambda$ is the EM wavelength in free space; $d$ is the thickness of the sample.

To determine $\varepsilon$ and $\mu$ of the sample under investigation, first, the analytical solution of the system of Eq. (2) relative to variables $x$ and $y$ must be found, then wave number $k$ and impedance $Z$ of the sample are calculated by

$$
k=\frac{1}{d} \arccos \left(\frac{x-y}{2}\right), \quad Z=\frac{y+\cos \varphi}{i \sin \varphi} .
$$

After performing calculations with Eq. (3), the permittivity and permeability of the sample are found by solving

$$
\varepsilon=\frac{c k}{(2 \pi f Z)}, \quad \mu=\frac{c k Z}{(2 \pi f)},
$$

where $f=c / \lambda$ is the frequency of the EMW.

The measurement bench is constructed using a four-port vector network analyzer, ZVA40. Two sets of transmitting or receiving wideband-lens horn antennas operating at subbands 3-24 and 22-39 GHz are used for measurements. A schematic diagram of the measurement bench is presented in Fig. 7. Since the linear dimensions of the measured samples are smaller than the size of the aperture of

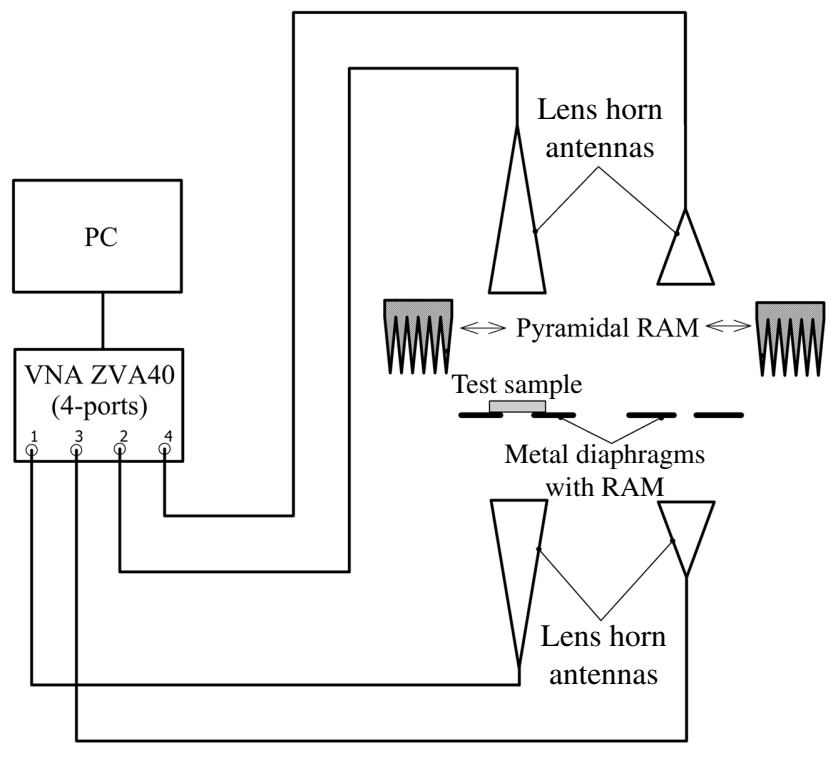

FIG. 7. Block diagram of measurement bench.

the antennas, the aperture is diaphragmed using specially designed circular metal diaphragms of various sizes with a broadband RAM coating.

A test sample is placed at the aperture of the metal diaphragm positioned in the near field of the lens horn antenna to minimize the impact of diffraction at the edges of the test sample and at the edges of the horn antenna. When measuring the reflection coefficient, a matched load is mounted in the area behind the diaphragm, namely, a RAM in the form of a three-dimensional pyramid-type block with a low value of reflectivity in the operating bandwidth (at least, $-50 \mathrm{~dB}$ ), to exclude undesirable parasitic reflections from the surroundings. To reduce the measurement error of the $S$ parameters of the test samples, a set of special calibrations with an echo cancellation is used.

Due to the length and imperfection of the measuring path (roughly $10 \mathrm{~m}$, including measuring cables), several peaks are introduced into the measured values due to rereflections between the inhomogeneities present in the measuring path. To overcome this, a clutter-reflection time-domain analysis is performed during digital-signal processing to eliminate these multiple rereflections. In addition, digital-processing methods are applied to suppress signal oscillations at the edges of the frequency range caused by the Gibbs phenomenon [29,30].

Images of a test sample for measuring complex permittivity and permeability in free space are shown in Fig. 8: the low-frequency section of the bench is shown in Fig. 8(a) and the high-frequency section is shown in Fig. 8(b).

The calibration procedure for reflection measurements $\left(S_{11}^{M}\right)$ consists of two different measurements: $R_{\text {bgr }}$ measures the reflection signal from the pure diaphragm loaded with pyramidal absorbing material (used in anechoic 
(a)

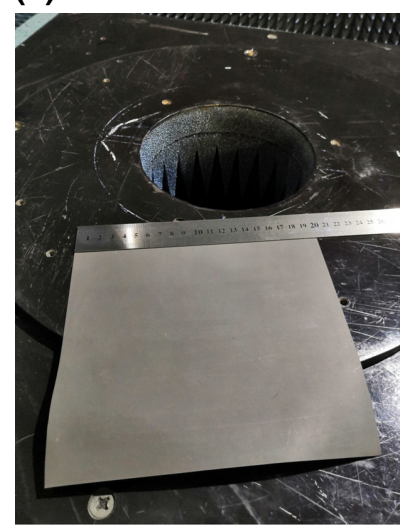

(b)

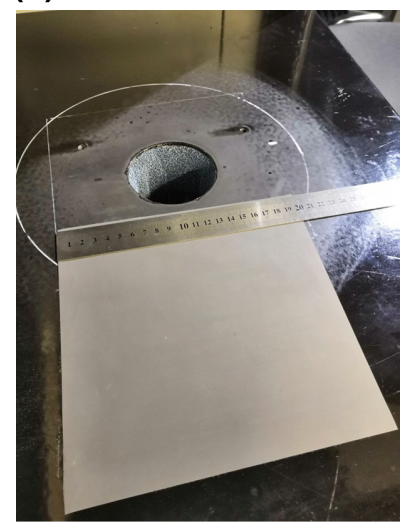

FIG. 8. Sample under investigation for measurements of complex permittivity and permeability in free space: (a)lowfrequency segment of the bench, (b) high-frequency segment of the bench.

chambers) with a reflectivity of less than $-50 \mathrm{~dB}$ over the operating frequencies, and $R_{\mathrm{rsp}}$ measures the reflection signal from the metal polished plate being tested (reflectivity equals $100 \%$ ). The calibration procedure for transmission measurements $\left(S_{21}\right)$ consists of two other measurements: $T_{\text {bgr }}$ measures the transmission signal through the diaphragm loaded by a metal plate coated with magnetic absorbing material to minimize radiation seepage through the gap between the diaphragm and plate, and $T_{\text {rsp }}$ measures the transmission signal through a pure diaphragm (transmission is equal to $100 \%$ ). After measuring the reflection signal, $R$ and transmission signal, $T$ of the sample under investigation, the scattering parameters of the sample can be retrieved from the results of the near-field measurements:

$$
\begin{aligned}
& S_{11}^{M}=\frac{\left(R-R_{\mathrm{bgr}}\right)}{\left(R_{\mathrm{rsp}}-R_{\mathrm{bgr}}\right)}, \\
& S_{21}=\frac{\left(T-T_{\mathrm{bgr}}\right)}{\left(T_{\mathrm{rsp}}-T_{\mathrm{bgr}}\right)} .
\end{aligned}
$$

These calibration procedures with background subtractions, using phase-correcting lenses in the horn antennas; relative measurements; and accurate positioning of the sample on the diaphragm allow us to obtain quasiplane fronts of EMWs in the near field of the diaphragms and then apply basic Eq. (2), which is valid for plane waves. The accuracy of the reflection and transmission measurements of the samples under investigation in free space can be demonstrated by the frequency dependencies of reflection and transmission coefficients (modulus and phase) of the 3.45-mm-thick test quartz-glass plate in the frequency range from 3 to $39 \mathrm{GHz}$, as presented in Figs. 9 and 10, correspondingly. The calculation of the scattering parameters

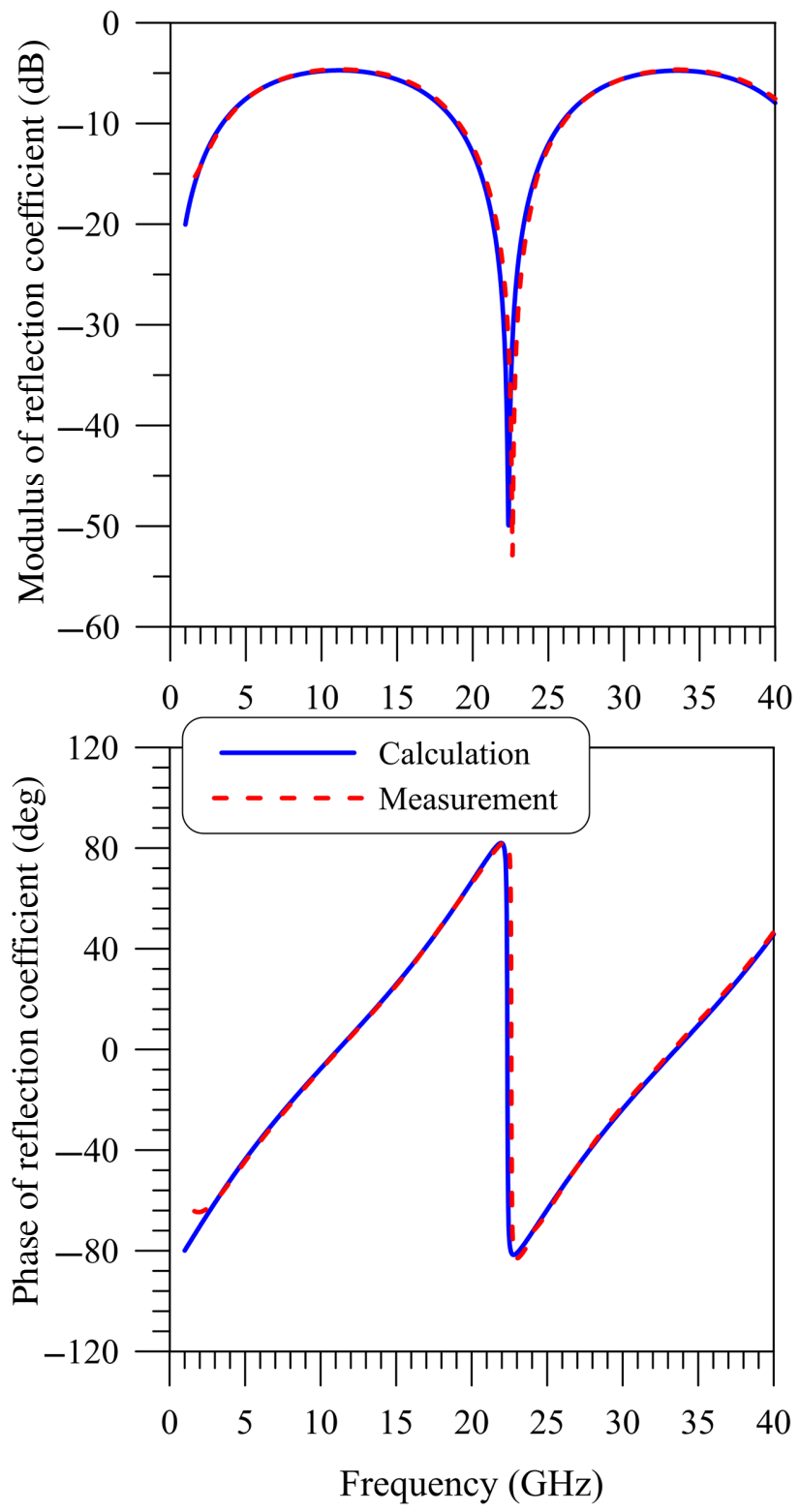

FIG. 9. Calculated (solid lines) and measured (dashed lines) frequency dependences of the modulus and phase of the reflection coefficient of the test quartz-glass plate.

for quartz glass is made for the well-known reference values for fused quartz $\left(\varepsilon^{\prime}=3.80 ; \varepsilon^{\prime \prime} \approx 0\right.$ in the microwave band) by using Eq. (2). The calculated values of the reflection (transmission) coefficient of the test quartz glass are presented as solid lines; the measured values are given as dashed lines.

The results presented in Figs. 9 and 10 demonstrate that calculated and measured data are close to each other. Therefore, the high accuracy of the measurement of scattering parameters for the sheet samples using the freespace method in the frequency range from 3 to $39 \mathrm{GHz}$ is proven. 

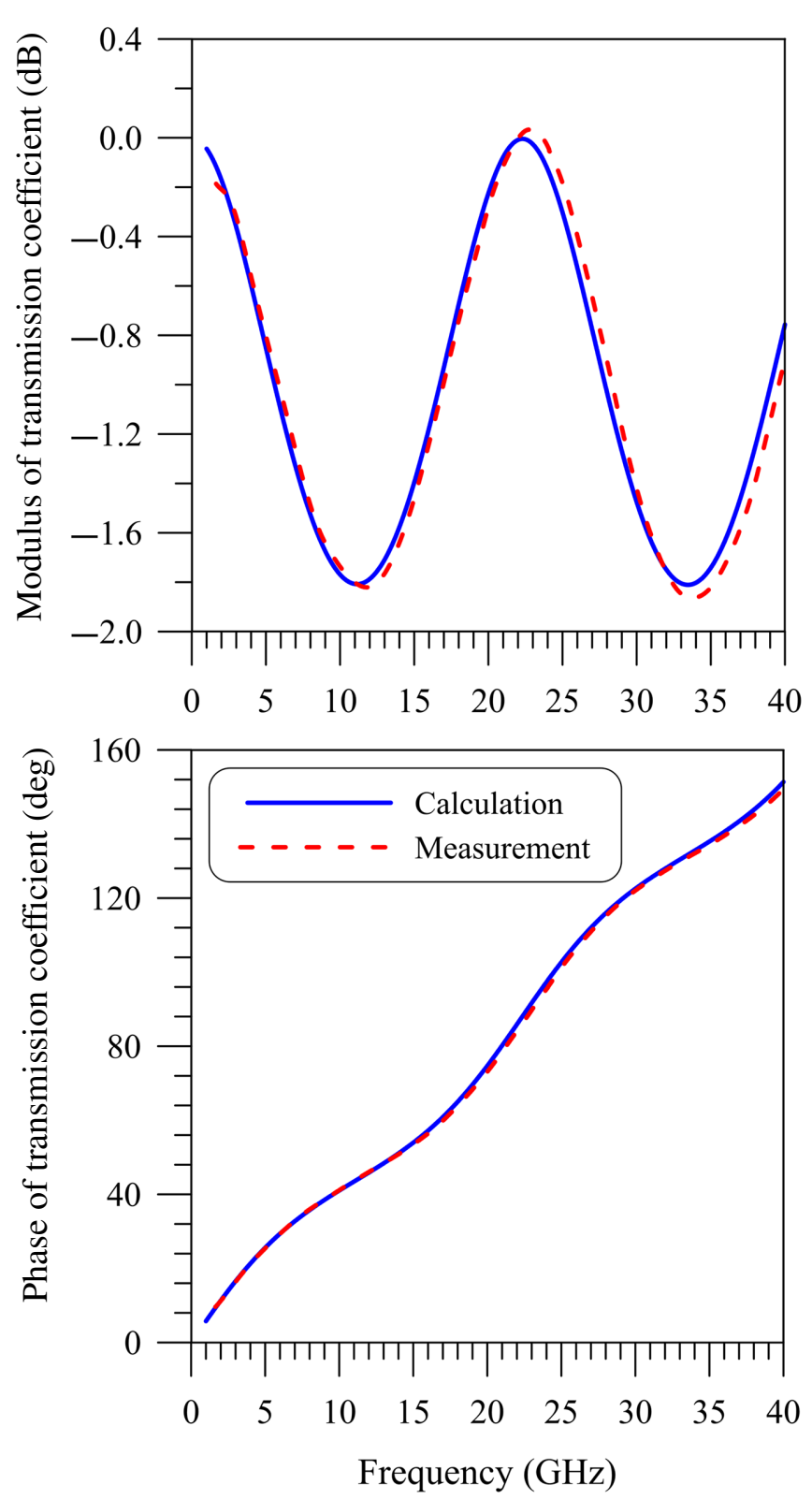

FIG. 10. Calculated (solid lines) and measured (dashed lines) frequency dependences of the modulus and phase of the transmission coefficient of the test quartz-glass plate.

The frequency dependence of the relative complex permittivity of the test sample of quartz glass is obtained from Eqs. (2)-(4) using the measured results of the frequency dependence of the transmission coefficient of quartz glass given in Fig. 10. The measured frequency dependences of the real and imaginary parts of the relative permittivity of test quartz glass in the frequency range from 3 to $39 \mathrm{GHz}$ are presented in Fig. 11.

The squares on the graph in Fig. 11 correspond to measurement results; the solid straight line corresponds to the reference values for fused quartz. The measurement error of the real part of quartz permittivity is less than $3 \%$;

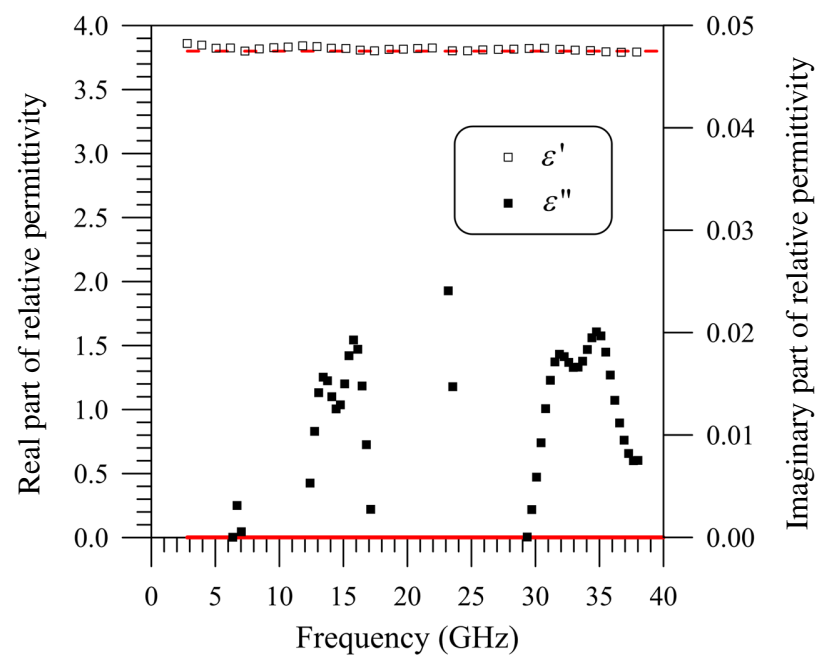

FIG. 11. Measured frequency dependences of the real and imaginary parts of the relative permittivity of test quartz glass over the frequency range from 3 to $39 \mathrm{GHz}$.

the measurement error of the imaginary part of the material permittivity without losses is significant (however, the average value is $\varepsilon^{\prime \prime} \approx 0$ ), but such a material is not under investigation in this study.

A second technique, using a coaxial line cell with a cross section of inner diameter $3.04 \mathrm{~mm}$ and outer diameter $7.00 \mathrm{~mm}$, is used to measure the complex permeability of samples under investigations from 0.1 to $5 \mathrm{GHz}$. Samples for the coaxial line cell are made of the same thin films as those used in the free-space method. To fill the cell as much as possible, the sample shape is a disk about $0.5 \mathrm{~mm}$ in thickness with a hole in its central part, which fits very tightly to the coaxial line. A diagram of the coaxial line cell is presented in Fig. 12.

The permeability of each sample is calculated by using the standing-wave method [31] on the results of measurements of the reflection coefficient, $R_{0}$, of the sample in short-circuit mode, which is described in more detail in Ref. [32]. The relationship between $R_{0}$ and the permeability, $\mu$, of the sample with thickness $d$ is expressed

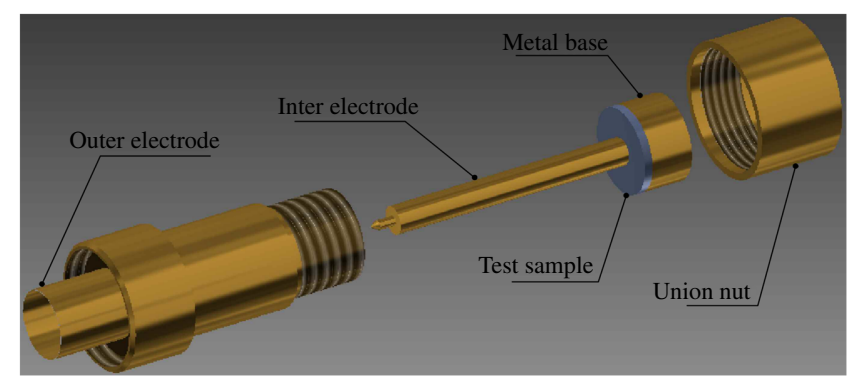

FIG. 12. Schematic of the coaxial line cell. 
by $[30]$

$$
R_{0}=\frac{(1-2 \pi i d \mu / \lambda)}{(1+2 \pi i d \mu / \lambda)} .
$$

It is worth noting that, in spite of utilizing the calibration of the measuring path, the coaxial method of measuring the material's parameters has one very significant potential systematic error. This is the possibility of air gaps between the outer and inner electrodes and the sample. Such gaps, in which the electrical field may be concentrated (because of the continuity of the normal component of electric induction) leads to systematic and unknown underestimates of the determined permittivity. Due to this issue, the frequency dependence of the complex permittivity of the samples obtained from measurements with the coaxial line cell are not given here.

Notably, when measuring the permeability of the sample, these errors do not occur because the presence of small air gaps does not lead to a change in the distribution of the magnetic field around the sample. The magnetic field in the coaxial line is parallel to the walls and then the errors in measuring of the sample permeability are absent. Thus, the free-space values of permeability of the sample and those extracted from coaxial line measurements are close to each other over the common frequency range from 3 to $5 \mathrm{GHz}$.

\section{MEASURED RESULTS FOR COMPOSITES WITH A HIGH VOLUME LOADING OF CIP}

First, we present experimental results for composites containing CIP as a magnetic phase with a high volume loading (about 44.3\%), which is the limit for maintaining acceptable mechanical properties of the composites. The complex permittivity of the composites based on CIP EN, HL, EW, and R-100F-2 are shown in Fig. 13, where the squares are experimental data, and the solid and dashed lines are fits of the experimental data by Cole-Cole relaxation expressed by [33]

$$
\varepsilon(f)=\varepsilon_{\infty}+\frac{A}{\left[1+i\left(f / f_{0}\right)^{\gamma_{e}}\right]},
$$

where $\varepsilon_{\infty}$ is the relative optical permittivity, $A$ is the relaxation amplitude, $f_{0}$ is the relaxation frequency, and $\gamma_{e}$ is the dielectric relaxation parameter $\left(\gamma_{e} \approx 0.1, \ldots, 1.0\right)$.

The fitting parameters of the Cole-Cole relaxation for composites based on CIPs EN, HL, EW, and R-100F-2 are presented in Table I.

These results show that the permittivity of all composites is weakly dependent on the frequency over bandwidths of 3-39 GHz, indicating low dielectric losses, which are explained by the very low static conductivity of the samples (the sheet resistance is more than $10 \mathrm{M} \Omega / \square$ ) and the Kramers-Kronig relationship for an analytical complex function of permittivity [34,35].

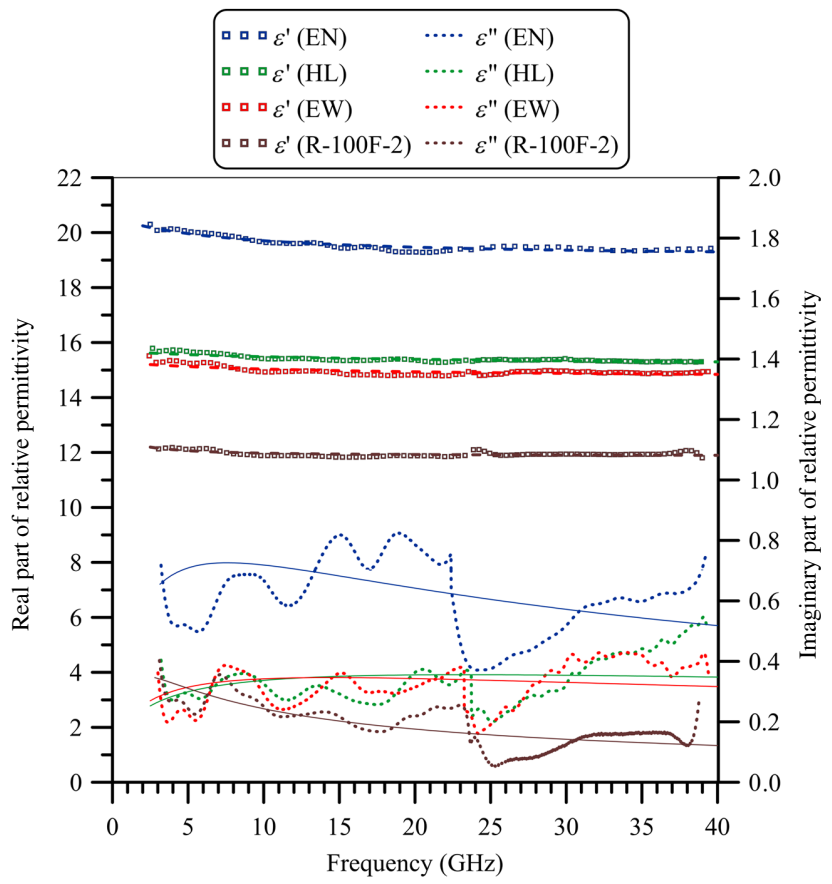

FIG. 13. Measured frequency dependence of the real and imaginary parts of the relative permittivity of composites based on CIP EN, HL, EW, and R-100F-2 for a volume loading of CIP of $44.3 \%$ in the frequency range from 3 to $39 \mathrm{GHz}$.

For the composite with the EN filler, which is characterized by the largest fraction (4-5- $\mu \mathrm{m}$ diameter particles) of the studied composites, the highest values of the real part of the relative permittivity of about 20 are observed. The imaginary part of the relative permittivity of all samples is no more than 0.9 and weakly depends on frequency.

In contrast to the permittivity, the permeability of the composites is characterized by a strong frequency dispersion over the frequency range of $0.1-39 \mathrm{GHz}$. The results for the real and imaginary parts of the relative permeability of composites are represented in Figs. 14 and 15. The dots indicate experimental data; the solid lines indicate a model dispersion dependence, which is the superposition of five magnetic Lorentz resonances [36]:

$$
\mu(f)=\mu_{\infty}+\sum_{k} \frac{B_{k} v_{k} f_{k}}{f_{k}^{2}-f^{2}-i v_{k} f} .
$$

TABLE I. Fitting parameters of experimental data for the relative permittivity of composites based on different CIP types (Fig. 13).

\begin{tabular}{lcccc}
\hline \hline CIP type & $\varepsilon_{\infty}$ & $A$ & $f_{0}(\mathrm{GHz})$ & $\gamma_{e}$ \\
\hline EN & 19.08 & 1.45 & 7.59 & 0.52 \\
EW & 14.64 & 0.69 & 14.40 & 0.42 \\
HL & 15.01 & 0.71 & 23.31 & 0.39 \\
R-100F-2 & 11.88 & 0.71 & 2.08 & 0.59 \\
\hline \hline
\end{tabular}




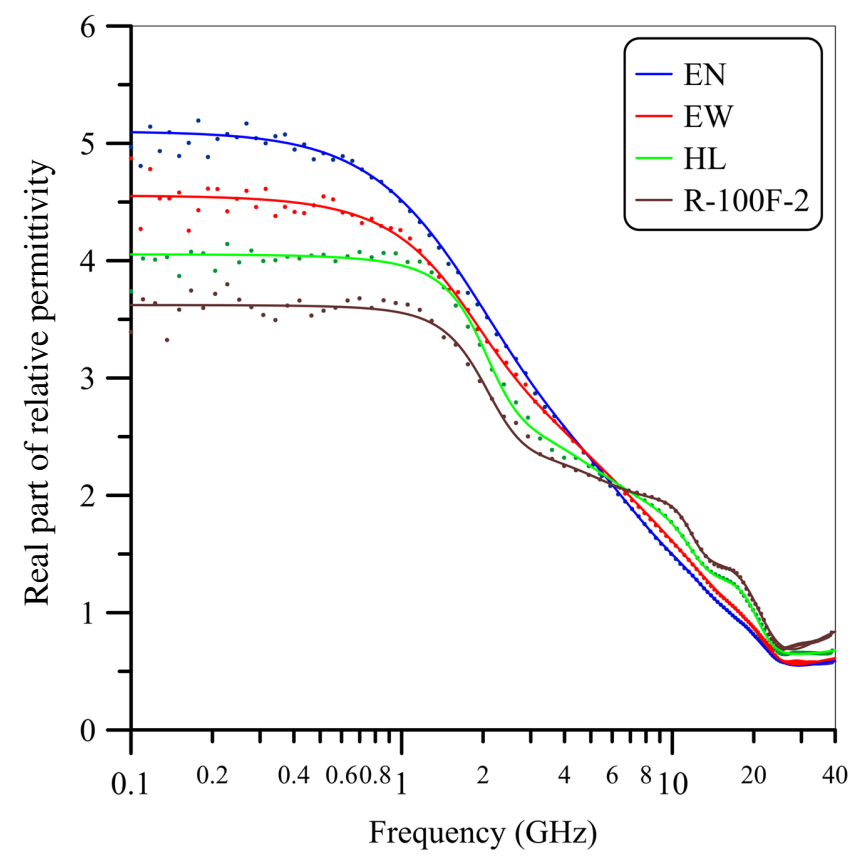

FIG. 14. Measured frequency dependence of the real part of the relative permeability of composites based on CIP EN, EW, $\mathrm{HL}$, and R-100F-2 for a volume loading of CIP of $44.3 \%$ over the frequency range from 0.1 to $39 \mathrm{GHz}$. Continuous lines are best fits using Eq. (6).

Here, $\mu_{\infty}$ is the optical relative permeability; $B_{k}, f_{k}$, and $v_{k}$ are the amplitudes, natural frequencies, and half-widths of the magnetic resonances; $k=1, \ldots, 5$. The fitting parameters of the magnetic Lorentz resonances for different types of CIP (Figs. 14 and 15) are presented in Table II. These parameters show that all composites based on different CIP types possess resonance peaks above $20 \mathrm{GHz}$ with a $Q$ factor above one.

It is known $[37,38]$ that the frequency dependence of permeability can be described by the following physical phenomena: domain-wall resonance (DWR), natural ferromagnetic resonance (FMR), and the skin effect in conducting inclusions. The relative contribution of these mechanisms to magnetic losses depends on the form, composition, and concentration of the inclusions, the frequency, and many other factors. Methods for quantifying the contributions of the various mechanisms to the effective permeability of a composite material have not been fully developed yet and are not discussed further here.

Looking at Figs. 14 and 15, one can see that for the composites based on CIPs EN and EW the frequency dependence of the imaginary part of the complex relative permeability shows a broad maximum, within which, at least for composites based on CIPs HL and R-100F-2, there are three clear resonant features. The primary maximum is at about $2 \mathrm{GHz}$ (see Table I). This is caused by the skin effect in magnetic inclusions [38]. It is the skin effect that forces the maximum of the magnetic losses to

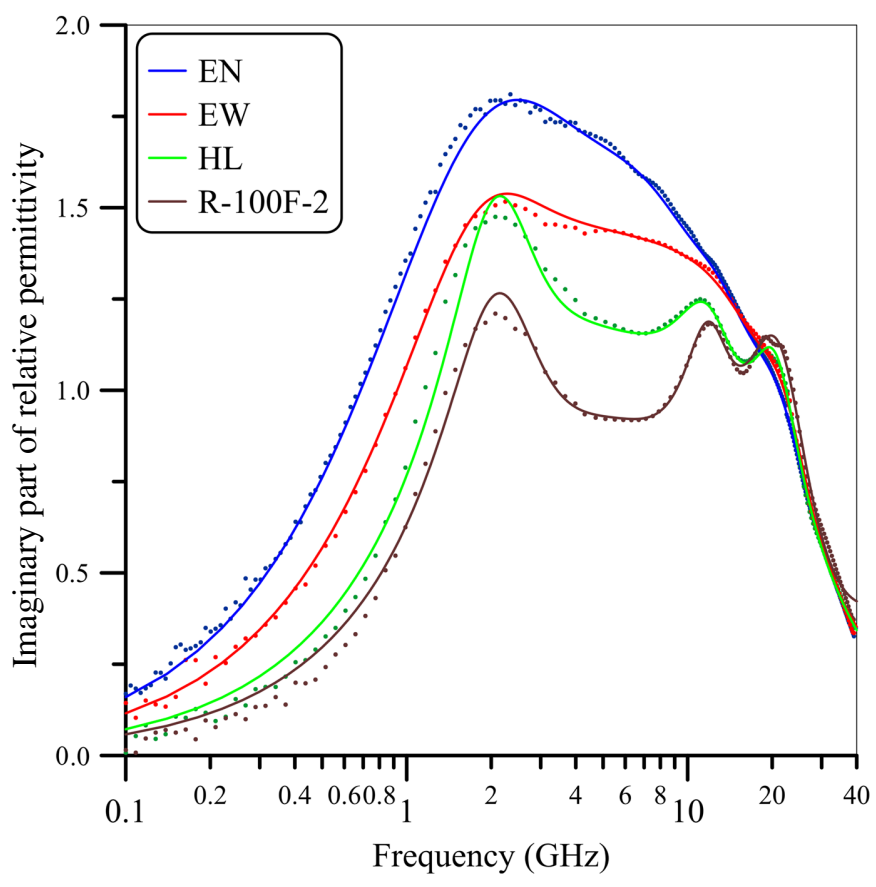

FIG. 15. Measured frequency dependence of the imaginary part of relative permeability of composites based on CIP EN, EW, HL, and R-100F-2 for a volume loading of CIP of $44.3 \%$ over the frequency range from 0.1 to $39 \mathrm{GHz}$. Continuous lines are best fits using Eq. (6).

be located not at the FMR frequency, but at frequencies where the minimum size of the inclusions (for spherical particles, in our case, it is the diameter of the grain) is equal to the thickness of the skin layer. As a result of the spread in particle sizes within the samples, this frequency peak of magnetic losses is broadened substantially, particularly for the larger particle sizes of composites based on CIPs EN and EW. Of all studied fillers, CIPs EN and EW are characterized by both the largest grain size and a very wide particle size distribution.

It should be noted that composite, filled with CIP EN, in addition to having the highest value of permittivity, also has the highest value of permeability (maximum values of static permeability and imaginary part of complex permeability in the frequency range under investigation) among all measured composites. This is because the magnetic filler EN has a maximum average granule size of about $4.5 \mu \mathrm{m}$ (Fig. 3).

Composites based on CIPs HL and R-100F-2 show more peaks in the frequency-dependent imaginary relative permeability. Granulometric analysis of these CIPs, together with measurements of the material parameters of composites based on them, shows that these fillers have similar EM properties and can be considered as analogues. However, it is worth noting that composite, based on CIP R-100F-2 (phosphated iron powder), has the lowest value of relative permittivity among all samples under study, namely, 
TABLE II. Fitting parameters of experimental data for the relative permeability of composites based on different CIP types (Figs. 9 and 10).

\begin{tabular}{lccccc}
\hline \hline CIP & $\mu_{\infty}$ & $k$ & $B_{k}$ & $f_{k}(\mathrm{GHz})$ & $v_{k}(\mathrm{GHz})$ \\
\hline EN & 0.90 & 1 & 0.91 & 3.28 & 7.17 \\
& & 2 & 0.63 & 9.22 & 14.88 \\
& & 3 & 0.19 & 12.72 & 9.77 \\
& & 4 & 0.31 & 22.19 & 14.91 \\
EW & & 5 & 0.31 & 32.61 & 104.01 \\
& 1.44 & 1 & 0.56 & 2.17 & 3.33 \\
& & 2 & 0.75 & 8.38 & 20.33 \\
& & 3 & 0.40 & 8.39 & 15.46 \\
& & 4 & 0.22 & 14.89 & 15.83 \\
HL & 5 & 0.33 & 22.55 & 13.83 \\
& 1.07 & 1 & 0.72 & 2.21 & 2.04 \\
& & 2 & 0.41 & 12.20 & 8.38 \\
& & 3 & 0.52 & 14.32 & 48.49 \\
& & 4 & 0.50 & 20.92 & 11.06 \\
R-100F-2 & 1.11 & 1 & 0.71 & 2.26 & 2.26 \\
& & 2 & 0.51 & 7.50 & 13.96 \\
& & 3 & 0.46 & 12.09 & 6.40 \\
& & 4 & 0.84 & 21.82 & 15.90 \\
& & 5 & 0.18 & 24.29 & 381.62 \\
\hline \hline
\end{tabular}

$\varepsilon^{\prime}=12$. This is due to the formation of a significant insulating film of iron phosphate on the particles during treatment of the feedstock iron powder with orthophosphoric acid.

\section{CONCENTRATION DEPENDENCE OF THE COMPLEX RELATIVE PERMITTIVITY AND PERMEABILITY OF COMPOSITES BASED ON CIP R-100F-2}

The frequency dependence of the complex relative permittivity and permeability of composites with different volume loadings of CIP R-100F-2, varying from 3.3 to $40.3 \%$, in a polymer binder are presented in Figs. 16 and 17.

From Fig. 16, it can be seen that $\varepsilon^{\prime}(f)$ and $\varepsilon^{\prime \prime}(f)$ obtained by measuring samples in free space over bandwidths of 3-39 GHz are weakly dependent on frequency. This frequency dependence is well approximated by ColeCole relaxation, Eq. (5).

The fitting parameters of the Cole-Cole relaxation for CIP R-100F-2 with different volume loadings (Fig. 16) are presented in Table III.

In contrast, the frequency dependences of the microwave relative permeability of the composites under study, as shown in Figs. 17 and 18, are characterized by significant dispersion, with five distinct resonance modes. The low-frequency resonance mode is a homogeneous FMR. If the carbonyl iron grains are homogeneous spheres, then, in accordance with Snoek's law, Eq. (1), the frequency of the

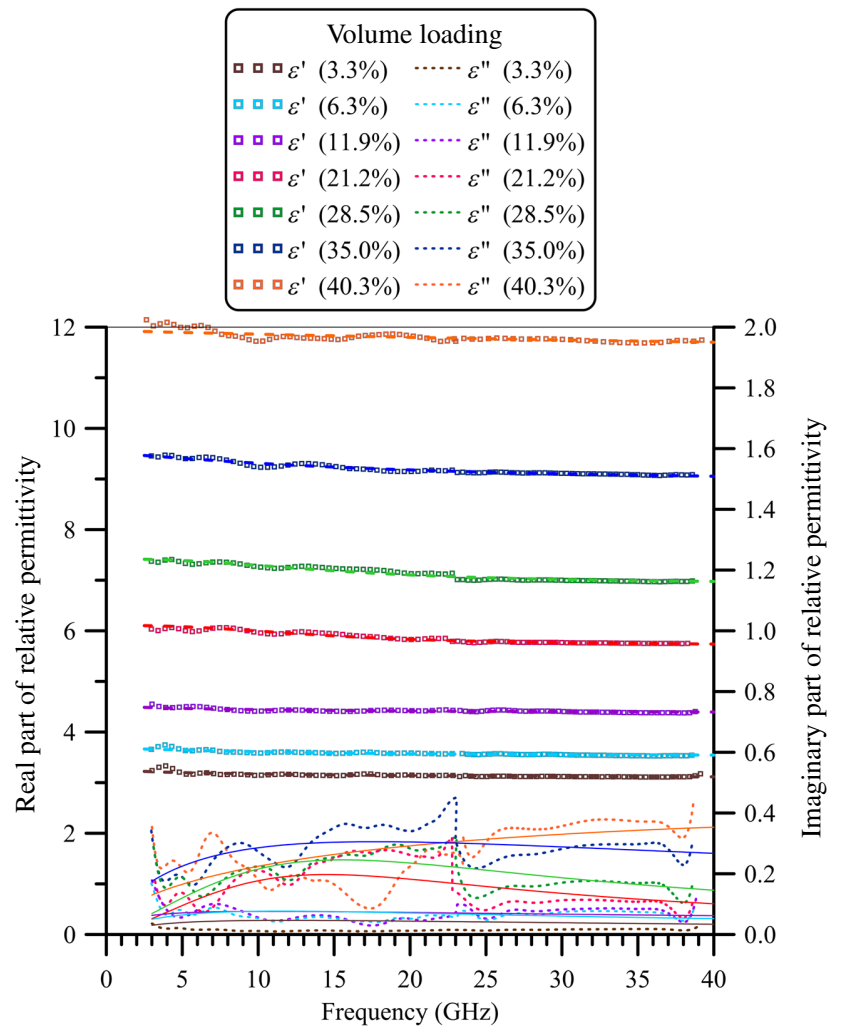

FIG. 16. Relative complex permittivity of composites based on CIP R-100F-2 with increasing volume loading of CIP over the frequency range from 3 to $39 \mathrm{GHz}$.

natural FMR should be in the megahertz range; estimates give values of the FMR frequency in the range from 200 to $600 \mathrm{MHz}$. However, since carbonyl iron particles constitute a complex layered structure, in which the Fe grains form highly anisotropic shells, alternating with thin dielectric layers, the resonance frequency of the FMR is shifted to higher frequencies and takes a value of about $2 \mathrm{GHz}$. High-frequency modes of carbonyl iron are at frequencies exceeding the frequency of the homogeneous FMR and are characteristic of homogeneous magnetically isolated particles, the magnetic moments of which are not correlated with each other.

The fitting parameters of the magnetic Lorentz resonances for CIP R-100F-2 with different volume loadings (Figs. 17 and 18) are presented in Table IV.

From Fig. 17, it can be seen that all curves for the real part of relative permeability cross at nearly the same so-called cross frequency, $f_{c}$, located between 21 and $22 \mathrm{GHz}$, independently of the volume loading of CIP. This cross frequency, $f_{c}$, can be extracted from the fundamental Kramers-Kronig relationship [39] (causality principle) for composite permeability:

$$
\mu^{\prime}\left(f_{c}\right)=1+\left(\frac{1}{\pi}\right) \int_{0}^{\infty}\left[\frac{\mu^{\prime \prime}(f)}{\left(f-f_{c}\right)}\right] d f \approx 1.1
$$




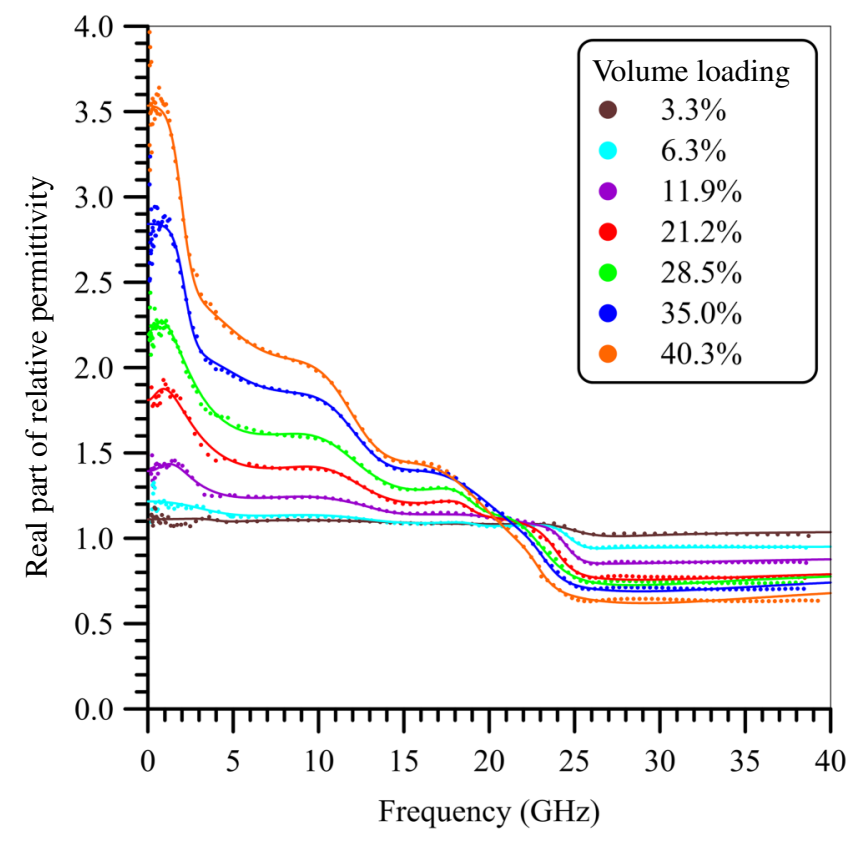

FIG. 17. Frequency dependence of the real part of the complex relative permeability of composites based on CIP R-100F-2 with increasing volume loading of CIP over the frequency range from 0.1 to $39 \mathrm{GHz}$.

The numerical solutions of Eq. (7) for different loadings of CIP reveal that the frequency $f_{c}$ is distributed over a small range from 21 to $22 \mathrm{GHz}$, independent of the volume loading of CIP. The important fact is that $f_{c}<f_{5}$ (see Table II) for all CIP loadings.

High-frequency modes of carbonyl iron are described in Refs. [12,36,40]. Ref. [36] presents a physical model of carbonyl iron, in which high-frequency resonance modes are proposed to be considered as Aharoni exchange [41] modes.

The excitation of exchange modes in isolated particles can arise when at least one geometric dimension approaches the nanometer size range. The frequency, $\omega$, for the exchange resonance in spherical particles is given

TABLE III. Fitting parameters of experimental data for the relative permittivity of composites based on CIP R-100F-2 (Fig. 16).

\begin{tabular}{lcccc}
\hline \hline Volume loading $(\%)$ & $\varepsilon_{\infty}$ & $A$ & $f_{0}(\mathrm{GHz})$ & $\gamma_{e}$ \\
\hline 3.3 & 3.07 & 0.19 & 11.95 & 0.45 \\
6.3 & 3.53 & 0.15 & 11.42 & 0.75 \\
11.9 & 4.36 & 0.15 & 10.62 & 0.50 \\
21.2 & 5.71 & 0.40 & 14.63 & 1.26 \\
28.5 & 6.92 & 0.49 & 15.82 & 1.21 \\
35.0 & 8.90 & 0.61 & 17.71 & 0.65 \\
40.3 & 11.45 & 0.61 & 26.49 & 0.43 \\
\hline \hline
\end{tabular}

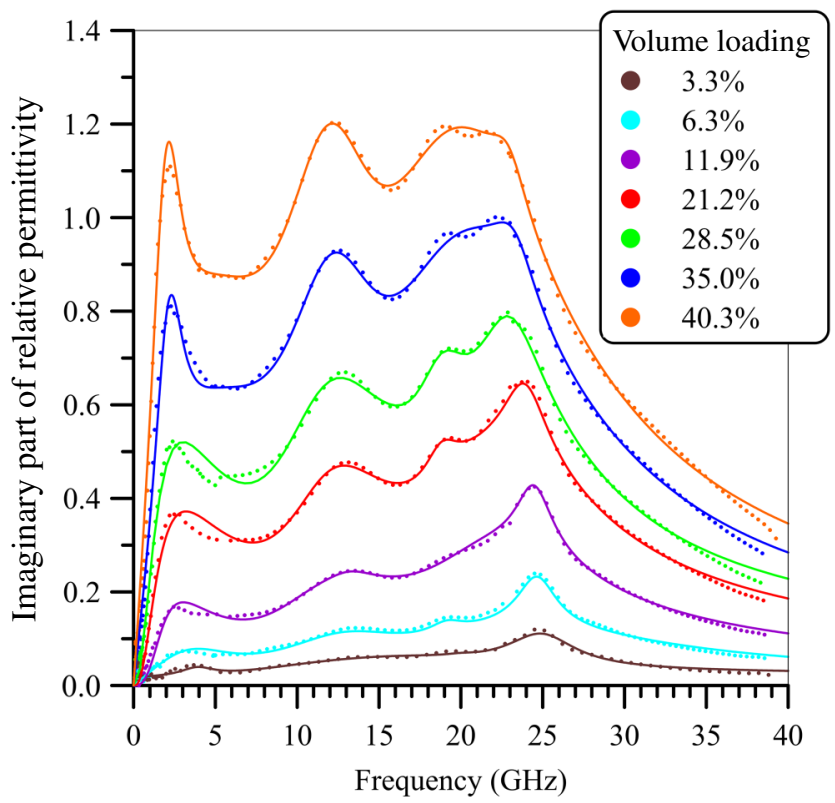

FIG. 18. Frequency dependence of the imaginary part of the relative permeability of composites based on CIP R-100F-2 with increasing volume loading of CIP over the frequency range from 0.1 to $39 \mathrm{GHz}$.

by [41]

$$
\omega=\gamma\left(\frac{C \mu_{k n}^{2}}{R_{2}^{2} M_{s}}+H_{z}\right)
$$

where $C$ is the exchange constant, $R_{2}$ is the outer radius, $\mu_{k n}$ are the eigenvalues, and $\gamma H_{z}$ is the ferromagnetic resonance frequency. For a magnetic spherical shell with inner radius $R_{1}$, applying the pinned boundary condition $\partial \mathbf{m}_{i} / \partial r+2 K_{S} \mathbf{m}_{i} / C=0$ on the outer surface, the eigenvalues, $\mu_{k n}$, are calculated from [42]

$$
\begin{aligned}
& {\left[\frac{\mu_{k n}}{R_{2}} \frac{\partial j_{n}\left(\mu_{k n}\right)}{\partial \mu_{k n}}+\frac{2 K_{s}}{C} j_{n}\left(\mu_{k n}\right)\right]\left[\frac{\partial y_{n}(\rho)}{\partial \rho}\right]_{\rho=\frac{\mu_{k n} R_{1}}{R_{2}}}} \\
& -\left[\frac{\mu_{k n}}{R_{2}} \frac{\partial y_{n}\left(\mu_{k n}\right)}{\partial \mu_{k n}}+\frac{2 K_{s}}{C} j_{n}\left(\mu_{k n}\right)\right]\left[\frac{\partial j_{n}(\rho)}{\partial \rho}\right]_{\rho=\frac{\mu_{k n} R_{1}}{R_{2}}}=0,
\end{aligned}
$$

where $i=x, y ; K_{s}$ is the surface anisotropy constant; and $j_{n}$ and $y_{n}$ are spherical Bessel functions of the first and second kind, respectively. In the limit of a very thin film or shell, it has been shown that the shape anisotropy reduces to a simple local anisotropy, which favours in-plane orientation of the magnetization [43]. In this situation, the shape and surface anisotropy are often difficult to distinguish experimentally, and constant $K_{s}$ can be treated as an effective magnetic anisotropy that incorporates the two anisotropy terms. The presence of pinning on the surface of the particle removes degeneracy between the lowest exchange 
TABLE IV. Fitting parameters of experimental data for the relative permeability of composites based on CIP R-100F-2 with different volume loadings (Figs. 17 and 18).

\begin{tabular}{|c|c|c|c|c|c|}
\hline $\begin{array}{l}\text { Volume } \\
\text { loading (\%) }\end{array}$ & $\mu_{\infty}$ & $k$ & $B_{k}$ & $f_{k}(\mathrm{GHz})$ & $v_{k}(\mathrm{GHz})$ \\
\hline 3.3 & 1.07 & $\begin{array}{l}1 \\
2 \\
3 \\
4 \\
5\end{array}$ & $\begin{array}{l}0.017 \\
0.021 \\
0.016 \\
0.004 \\
0.069\end{array}$ & $\begin{array}{c}4.08 \\
11.68 \\
17.75 \\
20.00 \\
25.04\end{array}$ & $\begin{array}{c}2.51 \\
15.32 \\
13.90 \\
2.38 \\
5.10\end{array}$ \\
\hline 6.3 & 1.05 & $\begin{array}{l}1 \\
2 \\
3 \\
4 \\
5\end{array}$ & $\begin{array}{l}0.054 \\
0.086 \\
0.024 \\
0.105 \\
0.108\end{array}$ & $\begin{array}{c}4.82 \\
13.82 \\
19.17 \\
23.00 \\
24.69\end{array}$ & $\begin{array}{c}7.28 \\
10.44 \\
2.54 \\
18.23 \\
2.94\end{array}$ \\
\hline 11.9 & 1.39 & $\begin{array}{l}1 \\
2 \\
3 \\
4 \\
5\end{array}$ & $\begin{array}{c}0.147 \\
0.131 \\
0.021 \\
0.20 \\
0.143\end{array}$ & $\begin{array}{c}2.04 \\
11.77 \\
13.25 \\
22.16 \\
24.46\end{array}$ & $\begin{array}{c}3.39 \\
9.83 \\
3.69 \\
12.23 \\
2.75\end{array}$ \\
\hline 21.2 & 1.81 & $\begin{array}{l}1 \\
2 \\
3 \\
4 \\
5\end{array}$ & $\begin{array}{c}0.27 \\
0.27 \\
0.079 \\
0.29 \\
0.16\end{array}$ & $\begin{array}{c}1.64 \\
12.19 \\
18.81 \\
22.40 \\
23.90\end{array}$ & $\begin{array}{c}3.62 \\
8.02 \\
2.57 \\
12.11 \\
3.32\end{array}$ \\
\hline 28.5 & 2.20 & $\begin{array}{l}1 \\
2 \\
3 \\
4 \\
5\end{array}$ & $\begin{array}{l}0.36 \\
0.40 \\
0.18 \\
0.25 \\
0.21\end{array}$ & $\begin{array}{c}1.41 \\
11.96 \\
18.87 \\
22.90 \\
23.56\end{array}$ & $\begin{array}{c}3.37 \\
8.83 \\
3.78 \\
3.37 \\
12.04\end{array}$ \\
\hline 35.0 & 1.40 & $\begin{array}{l}1 \\
2 \\
3 \\
4 \\
5\end{array}$ & $\begin{array}{l}0.46 \\
0.39 \\
0.58 \\
0.78 \\
0.13\end{array}$ & $\begin{array}{c}2.41 \\
7.47 \\
12.69 \\
19.80 \\
23.30\end{array}$ & $\begin{array}{c}2.18 \\
12.59 \\
7.55 \\
13.72 \\
3.41\end{array}$ \\
\hline 40.3 & 1.49 & $\begin{array}{l}1 \\
2 \\
3 \\
4 \\
5\end{array}$ & $\begin{array}{l}0.65 \\
0.54 \\
0.72 \\
0.95 \\
0.12\end{array}$ & $\begin{array}{c}2.28 \\
7.55 \\
12.50 \\
19.41 \\
22.96\end{array}$ & $\begin{array}{c}2.26 \\
13.36 \\
7.45 \\
13.66 \\
2.89\end{array}$ \\
\hline
\end{tabular}

mode, $\mu_{10}$, and the uniform ferromagnetic resonance and increases the sensitivity of the exchange modes to variations in shell size and thickness. When the internal energies favor highly nonuniform magnetization states, it has been shown that multidomain magnetization configurations can also play an important role in broadening EMW absorption [44]. Nonuniform magnetization configurations are shown to exhibit multiple high-frequency modes that can reach frequencies of $20 \mathrm{GHz}$ for $\mathrm{Fe}$ shells [45,46] and can potentially manifest as several high-frequency resonance bands in the microwave absorption spectrum.

Figure 19 shows the dependence of the natural logarithm of the static relative permittivity (indicated in the

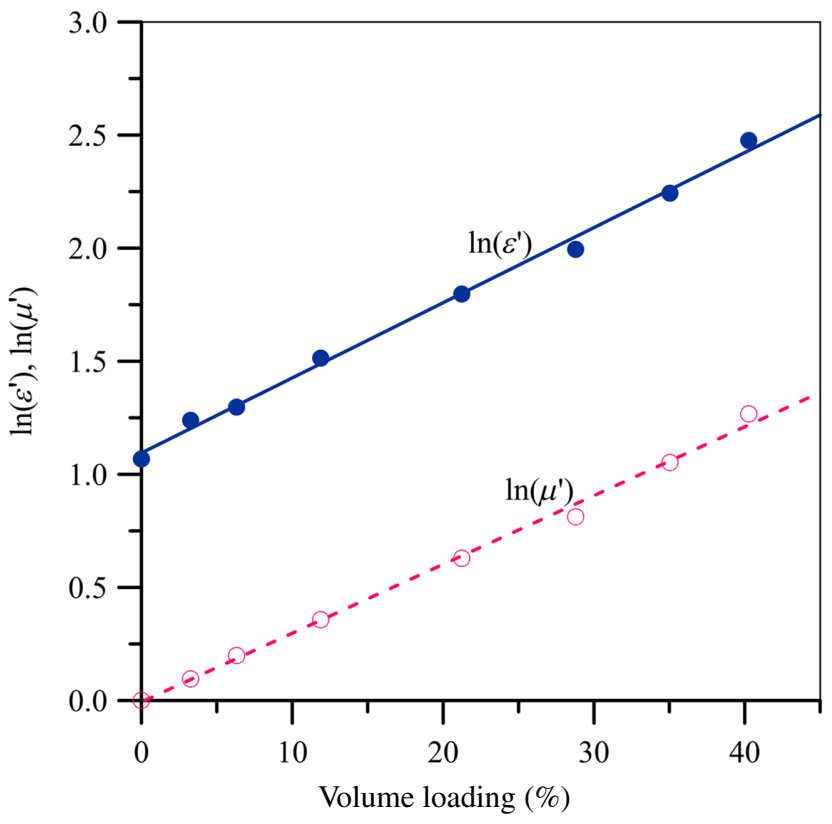

FIG. 19. Natural logarithm of static relative permittivity and permeability as a function of percentage volume loading for composites based on CIP R-100F-2.

graph by number 1) and permeability (indicated by number 2) of composites as a function of the volume loading of CIP R-100F-2. The logarithmic increase in the static relative permittivity and permeability of composites with increasing filler volume loading is in good agreement with Lichtenecker's law for a mixture of two components [47]. The values of parameters $\varepsilon^{\prime}=2.82$ and $\mu^{\prime}=1$ at a zero concentration of CIP in the composite material are obtained directly by the free-space method, when measuring the sample in the form of a thin polymer film. Points on the graphs correspond to experimental results; solid and dotted lines are linear approximations of the experimental results.

\section{RADAR-ABSORBING PROPERTIES OF COMPOSITE MATERIALS BASED ON CIP R-100F-2}

For a more detailed exploration of the radar-absorbing properties of CIP R-100F-2, with particular regard to its practical application, we calculate the frequency dependence of normal-incidence reflectivity for a number of composites with different volume loadings of the filler deposited onto a metal substrate. These calculations are based on the measurements of the dielectric and magnetic parameters given in Sec. V.

In this case, the practical task is to develop a RAM with a reflection coefficient below $-20 \mathrm{~dB}$, with the ability to control the frequency of the minimum but maintain the depth of the peak. Numerical simulations show that, to get such effect, one has to change both key parameters: the 
CIP concentration and the RAM thickness. By changing only the concentration of CIP, the frequency dependence can be achieved; however, the depth of the absorption peak will not be preserved. It is found that, using the original material, CIP R-100F-2, it is possible to maintain such a low absorption depth of the reflection coefficient in the frequency range of 10-30 GHz, with only minimal variation to the thickness, not exceeding $1.5 \mathrm{~mm}$. In our opinion, this is quite an important practical result. For CIP particles of other types, a similar criterion for the choice of concentrations and thicknesses led to different frequency bands. Next, we will show how the calculations are carried out.

According to the theory of long electrical lines, the input impedance, $Z_{\text {in }}$, of a single-layer metal-backed radarabsorbing material with impedance $Z=\sqrt{\mu / \varepsilon}$ and thickness $d$ under normal incidence of an EMW is given by

$$
Z_{\text {in }}=-i Z \tan (k d) .
$$

The modulus of the reflection coefficient, $R_{m}$, of a metalbacked magnetodielectric sample for a normal incidence of a plane EMW is written as

$$
R_{m}=20 \log _{10}\left|\frac{\left(Z_{\text {in }}-1\right)}{\left(Z_{\text {in }}+1\right)}\right| .
$$

The calculated frequency dependences of the normalincidence reflectivity of various thicknesses of composites filled with CIP R-100F-2 of different volume concentrations are shown in Fig. 20.

For the case of an ideal RAM with perfect matching, $Z=1$, the optimum values of thickness of composites are also determined, at which the deep minimum of reflectivity at the matching frequency is achieved. For practical reasons, the lower limit of the vertical axis on the graph in Fig. 20 is limited to $-50 \mathrm{~dB}$. The RAM thickness, $d$, is optimized in numerical simulations by minimizing the following function:

$$
\delta=d+5000 \Delta
$$

where $\Delta=\min \left(R-R_{d}\right), R_{d}$ is the designed modulus of the reflection coefficient, and $R$ is a minimum of the modulus of the reflection coefficient. Minimization is carried out using the standard Nelder-Mead algorithm (simplex method) [48].

As shown in Fig. 20, a shift of the absorption peaks in the low-frequency spectrum to the matching frequency of $10.6 \mathrm{GHz}$ is observed with increasing percentage volume loading of CIP R-100F-2 in a polymer binder. A matching frequency of $10.6 \mathrm{GHz}$ corresponds to the maximum CIP filling of $42.6 \%$. On the contrary, with a decrease in the filler concentration, a shift in the millimeter wavelength

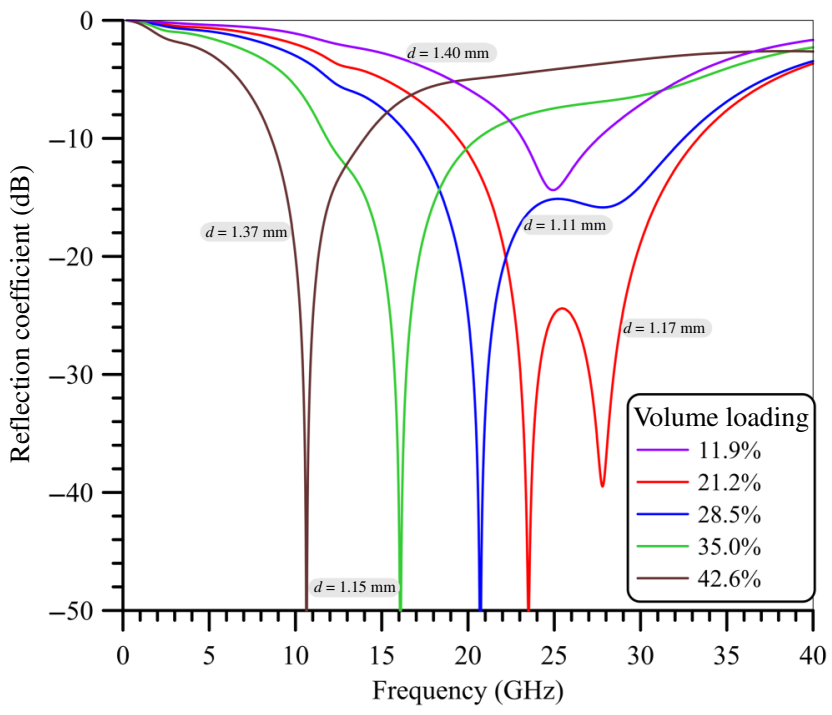

FIG. 20. Frequency dependence of the normal-incidence reflectivity of metal-backed composites with increasing percentage volume loading of CIP R-100F-2 with different thicknesses.

range is observed. With a decrease in volume loading of the filler to $21.2 \%$, one can see a tendency to form two minima of reflectivity (curve 2 in Fig. 20). Numerical simulations show that, with a further decrease in the concentration of CIP, it is impossible to obtain an equally deep minimum of the reflection coefficient. For a volume loading of $12 \%$,

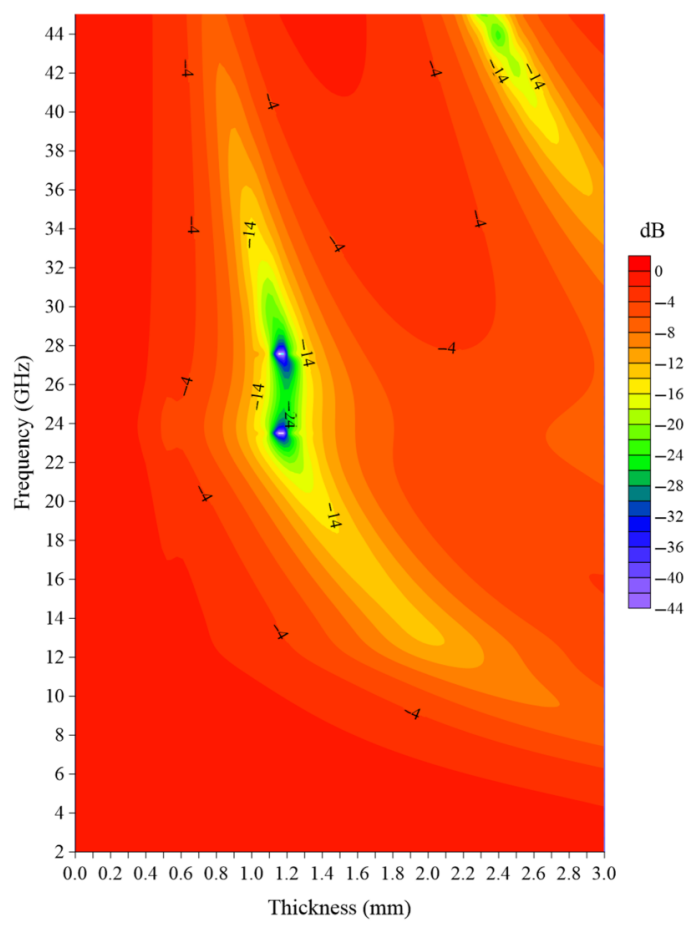

FIG. 21. Two-dimensional dependence of the normalincidence reflectivity of metal-backed composites with CIP $\mathrm{R}-100 \mathrm{~F}-2$ filling of $21.2 \%$. 
achieved by varying the RAM thickness from 1 to $2 \mathrm{~mm}$, it is possible to provide the lowest value of reflectivity of $-14 \mathrm{~dB}$ in terms of magnitude (see curve 1 in Fig. 20).

Figure 21 demonstrates the two-dimensional dependences of the normal-incidence reflectivity of metalbacked composites with CIP R-100F-2 filling of $21.2 \%$ on the frequency of the EMW and the thickness of the sample.

Perfect matching for a single-layer quarter-wavelength absorber with CIP R-100F-2 filling of $21.2 \%$ (Fig. 21 and curve 2 in Fig. 20; two deep minima of the reflection coefficient at resonant frequencies $f_{1}=23.61 \mathrm{GHz}$ and $f_{2}=27.84 \mathrm{GHz}$ ) is due to the proper frequency dispersion of real and imaginary parts of permeability at frequencies above the cross frequency, $f_{c}$. In other words, the real part of relative permeability is practically independent of frequency, and the imaginary part of the relative permeability has a decreasing dependence with increasing frequency. The minimum values of the reflection coefficient $\left(R_{m} \approx 0\right)$ correspond to [49]

$$
\frac{\pi d\left(\varepsilon^{\prime} \mu^{\prime \prime}+\varepsilon^{\prime \prime} \mu^{\prime}\right)}{\lambda \mu^{\prime}}=1,
$$

which may be simplified to (in our case $\varepsilon^{\prime \prime} \approx 0$ )

$$
\frac{\pi d f \varepsilon^{\prime} \mu^{\prime \prime}}{c \mu^{\prime}}=1
$$

Equation (14) is true for both resonant frequencies $f_{1}$ and $f_{2}$ of the reflection coefficient, so $\varepsilon^{\prime}\left(f_{1}\right) \approx \varepsilon^{\prime}\left(f_{2}\right) \approx 5.8$ and

$$
\frac{f_{1} \mu^{\prime \prime}\left(f_{1}\right)}{\mu^{\prime}\left(f_{1}\right)} \approx \frac{f_{2} \mu^{\prime \prime}\left(f_{2}\right)}{\mu^{\prime}\left(f_{2}\right)}
$$

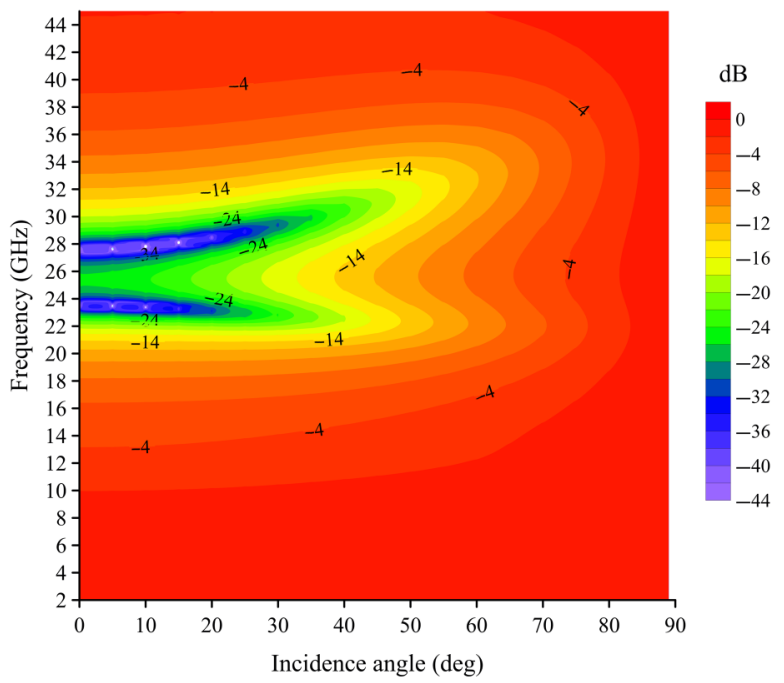

FIG. 22. Angle dependence of the reflectivity of metal-backed composites with CIP R-100F-2 filling of $21.2 \%$ for $s$-polarized radiation.

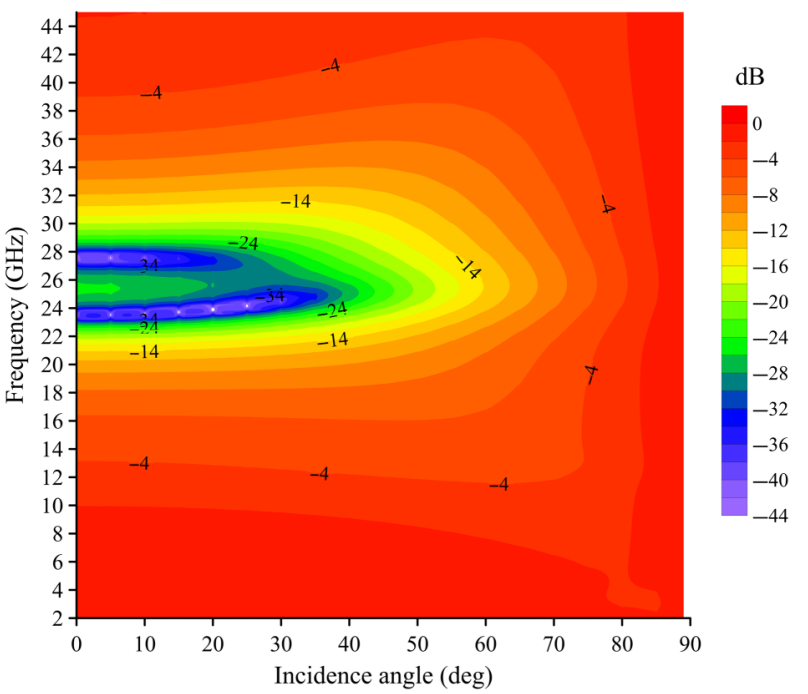

FIG. 23. Two-dimensional dependence of the reflectivity of metal-backed composites with CIP R-100F-2 filling of $21.2 \%$ for $p$-polarized radiation.

As a result, by using the model of a single-layer RAM with a relatively small thickness (approximately $1.2 \mathrm{~mm}$ ), it is shown that composite materials filled with CIP of type R-100F-2 with a volume loading of $21.2 \%$ can provide broadband matching (metal-backed reflection coefficient is less than $-20 \mathrm{~dB}$ ) in the frequency range from 22.3 to $29.8 \mathrm{GHz}$, that is, approximately $30 \%$ relative to a central frequency of $25.3 \mathrm{GHz}$.

Thus far, only the normal incidence of the electromagnetic wave on the composite RAM has been investigated; however, RAMs may be used under conditions of different illumination angles. The angular and frequency dependences of the reflection coefficient of a metal-backed composite sample filled with CIP of type R-100F-2 with a volume loading of $21.2 \%$ are presented in Fig. 22 for $s$-polarized radiation and in Fig. 23 for $p$-polarized radiation. This metal-backed composite sample possesses a low reflectivity of less than $-20 \mathrm{~dB}$ in both incidence planes for incidence angles of the EMW from normal to $40^{\circ}$ over a wide frequency range, approximately from 22 to $30 \mathrm{GHz}$.

\section{CONCLUSIONS}

The frequency-dependent complex relative permittivity and permeability of composite materials composed of an organosilicon polymer filled with fine fractions of CIP are measured by using two independent techniques that overlap in free space $(3-39 \mathrm{GHz})$ and in a coaxial line cell $(0.1-5 \mathrm{GHz})$.

The concentration dependences of the relative permittivity and permeability of composites filled with phosphated CIP R-100F-2 (average grain size is about $2 \mu \mathrm{m}$ ) are investigated. It is shown that the relative permeability of such 
composites has a multimodal structure, which is a superposition of FMR, DWR, and several high-frequency spinexchange resonances. The relative permittivity and permeability of the composites increase logarithmically with increasing percentage volume loadings of CIP; this agrees with Lichtenecker' law for a mixture of two components.

It is demonstrated that a single-layer RAM based on CIP of type R-100F-2 with a volume filling of $21.2 \%$ and a small thickness (no more than $1.2 \mathrm{~mm}$ ) on a metal plate gives a reflectivity minimum of less than $-20 \mathrm{~dB}$ over a wide frequency range from 22.3 to $29.8 \mathrm{GHz}$. This broadband minimum is approximately $30 \%$ with respect to the central operating frequency. This minimum is maintained for angles of incidence up to about $30^{\circ}$. These good absorption properties of the CIP composite over a wide frequency range and angles of incidence arise from both the anomalous variation of the permeability of the CIP composite for frequencies above the cross frequency, $f_{c}$, and the low value of composite permittivity and dielectric losses.

[1] X.-Y. Luo, W.-L. Guo, G. Ding, M. Du, K. Chen, J. Zhao, T. Jiang, and Y. Feng, Composite Strategy for BackwardScattering Reduction of a Wavelength-Scale Cylindrical Object by an Ultrathin Metasurface, Phys. Rev. Appl. 12, 064027 (2019).

[2] W. Jahjah, J.-P. Jay, Y. Le Grand, A. Fessant, A. R. E. Prinsloo, C. J. Sheppard, D. T. Dekadjevi, and D. Spenato, Electrical Manipulation of Magnetic Anisotropy in a $\mathrm{Fe}_{81} \mathrm{Ga}_{19} / \mathrm{Pb}\left(\mathrm{Mg}_{1 / 3} \mathrm{Nb}_{2 / 3}\right) \mathrm{O}_{3}-\mathrm{Pb}\left(\mathrm{Zr}_{\mathrm{x}} \mathrm{Ti}_{1-\mathrm{x}}\right) \mathrm{O}_{3}$ Magnetoelectric Multiferroic Composite, Phys. Rev. Appl. 13, 034015 (2020).

[3] N. I. Landy, S. Sajuyigbe, J. J. Mock, D. R. Smith, and W. J. Padilla, Perfect Metamaterial Absorber, Phys. Rev. Lett. 100, 207402 (2008).

[4] W. Zhang, S. Bie, H. Chen, Y. Lu, and J. Jiang, Electromagnetic and microwave absorption properties of carbonyl iron/ $\mathrm{MnO}_{2}$ composite, J. Magn. Magn. Mater. 358-359, 1 (2014).

[5] P. M. T. Ikonen, K. N. Rozanov, A. V. Osipov, P. Alitalo, and S. A. Tretyakov, Magnetodielectric substrates in antenna miniaturization: Potential and limitations, IEEE Trans. Antennas Propag. 54, 3391 (2006).

[6] L. Ferraris, F. Franchini, and E. Poskovic, in 2016 IEEE Sensors Applications Symposium (SAS), IEEE, Catania, Italy, (2016), pp. 1-6.

[7] V. G. Harris, Modern microwave ferrites, IEEE Trans. Magn. 48, 1075 (2012).

[8] F. Qin and C. Brosseau, A review and analysis of microwave absorption in polymer composites filled with carbonaceous particles, J. Appl. Phys. 111, 061301 (2012).

[9] A. Moya, D. Archilla, E. Navarro, A. Hernando, and P. Marín, Scattering of microwaves by a passive array antenna based on amorphous ferromagnetic microwires for wireless sensors with biomedical applications, Sensors 19, 3060 (2019).

[10] S. V. Shcherbinin, R. Perez, M. Vazquez, and G. V. Kurlyandskaya, Ferromagnetic resonance in electroplated
$\mathrm{CuBe} / \mathrm{FeCoNi}$ and amorphous $\mathrm{CoFeSiB}$ wires, IEEE Trans. Magn. 56, 1 (2020).

[11] N. Tian, J. W. Wang, F. Li, Z. Mei, Z. X. Lu, L. L. Ge, and C. Y. You, Enhanced microwave absorption of Fe flakes with magnesium ferrite cladding, J. Magn. Magn. Mater. 324, 4175 (2012).

[12] L. J. Deng, P. H. Zhou, J. L. Xie, and L. Zhang, Characterization and microwave resonance in nanocrystalline FeCoNi flake composite, J. Appl. Phys. 101, 103916 (2007).

[13] Y. Du, W. Liu, R. Qiang, Y. Wang, X. Han, J. Ma, and $\mathrm{P}$. $\mathrm{Xu}$, Shell thickness-dependent microwave absorption of core-shell $\mathrm{Fe}_{3} \mathrm{O}_{4} @ \mathrm{C}$ composites, ACS Appl. Mater. Interfaces 6, 12997 (2014).

[14] J. Wei, J. Liu, and S. Li, Electromagnetic and microwave absorption properties of $\mathrm{Fe}_{3} \mathrm{O}_{4}$ magnetic films plated on hollow glass spheres, J. Magn. Magn. Mater. 312, 414 (2007).

[15] B. Lu, X. L. Dong, H. Huang, X. F. Zhang, X. G. Zhu, J. P. Lei, and J. P. Sun, Microwave absorption properties of the core/shell-type iron and nickel nanoparticles, J. Magn. Magn. Mater. 320, 1106 (2008).

[16] J. L. Snoek, Dispersion and absorption in magnetic ferrites at frequencies above One Mc/s, Physica 14, 207 (1948).

[17] X. Liu, Z. Zhang, and Y. Wu, Absorption properties of carbon black/silicon carbide microwave absorbers, Composites, Part B 42, 326 (2011).

[18] J. Kim, S. Lee, and C. Kim, Comparison study on the effect of carbon nano materials for single-layer microwave absorbers in X-band, Compos. Sci. Technol. 68, 2909 (2008).

[19] T. Taniguchi, D. Saida, Y. Nakatani, and H. Kubota, Magnetization switching by current and microwaves, Phys. Rev. B 93, 014430 (2016).

[20] N. Hur, S. Park, P. A. Sharma, J. S. Ahn, S. Guha, and S.W. Cheong, Electric polarization reversal and memory in a multiferroic material induced by magnetic fields, Nature 429, 392 (2004).

[21] N. N. Phuoc, F. Xu, and C. K. Ong, Ultrawideband microwave noise filter: Hybrid antiferromagnet/ferromagnet exchange-coupled multilayers, Appl. Phys. Lett. 94, 092505 (2009).

[22] Q. Zeng, P. Chen, Q. Yu, H. Chu, X. Xiong, D. Xu, and Q. Wang, Self-assembly of ternary hollow microspheres with strong wideband microwave absorption and controllable microwave absorption properties, Sci. Rep. 7, 8388 (2017).

[23] H. Wang, N. Ma, Z. Yan, L. Deng, J. He, Y. Hou, Y. Jiang, and G. Yu, Cobalt/polypyrrole nanocomposites with controllable electromagnetic properties, Nanoscale 7, 7189 (2015).

[24] Q. Liu, Q. Cao, H. Bi, C. Liang, K. Yuan, W. She, Y. Yang, and R. Che, Coni@SiO $\mathrm{Si}_{2} @ \mathrm{TiO}_{2}$ and CoNi@Air@TiO microspheres with strong wideband microwave absorption, Adv. Mater. 28, 486 (2016).

[25] Y. Naito and K. Suetake, Application of ferrite to electromagnetic wave absorber and Its characteristics, IEEE Trans. Microwave Theory Tech. 19, 65 (1971).

[26] V. L. Volkov, V. G. Syrkin, and I. S. Tolmasskii, Carbonyl Iron (in Russian) (Metallurgiya, Moscow, 1969). 
[27] J. Grangle and G. M. Goodman, The magnetization of pure iron and nickel, Proc. R. Soc. London, Ser. A 321, 477 (1971).

[28] L. M. Brekhovskikh, Waves in Layered Media (Academic Press, NY, 1960).

[29] Cheh Pan, Gibbs phenomenon removal and digital filtering directly through the fast Fourier transform, IEEE Trans. Signal Process. 49, 444 (2001).

[30] V. N. Semenenko, V. A. Chistyaev, A. A. Politiko, and K. M. Baskov, Test stand for measuring the free-space electromagnetic parameters of materials over an ultrawide range of microwave frequencies, Meas. Tech. 62, 161 (2019).

[31] S. Roberts and A. Von Hippel, A new method for measuring dielectric constant and loss in the range of centimeter waves, J. Appl. Phys. 17, 610 (1946).

[32] K. N. Rozanov and N. A. Simonov, Microwave measurements of the magnetic film permeability, J. Commun. Technol. Electron. 47, 210 (2001).

[33] H. D. Choi, K. Y. Cho, S. Han, H. G. Yoon, and T. J. Moon, Frequency dispersion characteristics of the complex permittivity of the epoxy carbon black composites, J. Appl. Polym. Sci. 67, 363 (1998).

[34] H. A. Kramers, La diffusion de la lumiere par les atomes, Atti Congr Inten. Fis. Como 2, 545 (1927).

[35] R. Kronig, On the theory of dispersion of X-rays, J. Opt. Soc. Am. 12, 661 (1926).

[36] A. N. Lagarkov, V. N. Semenenko, V. A. Chistyaev, and I. T. Iakubov, High-Frequency modes in magnetic spectra of carbonyl iron, J. Magn. Magn. Mater. 324, 3402 (2012).

[37] A. V. Lopatin, Y. N. Kazantsev, N. E. Kazantseva, V. N. Apletalin, V. P. Mal'tsev, A. D. Shatrov, and P. Saha, Radio absorbers based on magnetic polymer composites and frequency-selective surfaces, J. Commun. Technol. Electron. 53, 1114 (2008).

[38] E. P. Elsukov, K. N. Rozanov, S. F. Lomaeva, A. V. Osipov, D. A. Petrov, S. N. Starostenko, A. S. Shuravin, A. L. Ulyanov, A. A. Chulkina, and D. V. Surnin, Influence of shape and chemical and phase compositions of ironcontaining particles on microwave performance of composites with an insulating matrix, Tech. Phys. 54, 569 (2009).

[39] L. D. Landau, E. M. Lifshitz, and L. P. Pitaevskii, in Electrodynamics of Continuous Media, 2nd ed., edited by E. M. Lifshitz and L. P. Pitaevskii (Pergamon Press, NY, 1984).

[40] D. Mercier, J.-C. S. Lévy, G. Viau, F. Fiévet-Vincent, F. Fiévet, P. Toneguzzo, and $\mathrm{O}$. Acher, Magnetic resonance in spherical Co-Ni and Fe-Co-Ni particles, Phys. Rev. B 62, 532 (2000).

[41] A. Aharoni, Exchange resonance modes in a ferromagnetic sphere, J. Appl. Phys. 69, 7762 (1991).

[42] C. McKeever, F. Y. Ogrin, and M. M. Aziz, Influence of surface anisotropy on exchange resonance modes in spherical shells, J. Phys. D: Appl. Phys. 51, 305003 (2018).

[43] Y. Gaididei, V. P. Kravchuk, and D. D. Sheka, Curvature Effects in Thin Magnetic Shells, Phys. Rev. Lett. 112, 257203 (2014).

[44] Z. Liu, R. Che, Y. Wei, Y. Liu, A. A. Elzatahry, D. A. Dahyan, and D. Zhao, Broadening microwave absorption via a multi-domain structure, APL Mater. 5, 046104 (2017).

[45] N. Vukadinovic and F. Boust, Three-dimensional micromagnetic simulations of multidomain bubble-state excitation spectrum in ferromagnetic cylindrical nanodots, Phys. Rev. B 78, 184411 (2008).

[46] C. McKeever, F. Y. Ogrin, and M. M. Aziz, Microwave magnetization dynamics in ferromagnetic spherical nanoshells, Phys. Rev. B 100, 054425 (2019).

[47] R. Simpkin, Derivation of Lichtenecker's logarithmic mixture formula from Maxwell's equations, IEEE Trans. Microwave Theory Tech. 58, 545 (2010).

[48] J. A. Nelder and R. Mead, A simplex method for function minimization, Comput. J. 7, 308 (1965).

[49] K. N. Rozanov and M. Y. Koledintseva, Matching conditions for a homogeneous absorbing layer, Procedia Eng. 216, 79 (2017). 